\title{
A Topological Invariant for Competitive Markets
}

by

Graciela Chichilnisky, Columbia University

March 1996 * revised April 1996

Discussion Paper Series No. 9596-15 


\title{
A Topological Invariant for Competitive Markets
}

\author{
Graciela Chichilnisky*
}

March 1996 revised April 1996

\begin{abstract}
I summarize and extend my results establishing that limited arbitrage is necessary and sufficient for the existence of a competitive equilibrium and for the compactness of the Pareto frontier [11] (announced in [14] and [8]). In response to a comment [36], I show that the authors misunderstood my results by focussing on brief announcements [14] and [8] which did not detail the hypothesis, rather than on the publication which contains the proofs [11]. The original results are extended here to encompass "mixed economies" based on [16]. They are used to define a topological invariant for a competitive market, which encodes exact information on its equilibria and on its social diversity, and predicts a failure of 'effective' demand.
\end{abstract}

\section{Introduction}

Limited arbitrage is a unifying concept for resource allocation. It is defined from the traders' endowments and preferences, and is simultaneously necessary and sufficient for the existence of a competitive equilibrium, ${ }^{1}$ for the nonemptiness of the core ${ }^{2}$ and for the existence of satisfactory social choice rules. ${ }^{3}$ This paper responds to a recent comment on my work on market equilibrium [36]. The three authors misunderstood my results by focussing on brief announcements in papers which cover other areas, algebraic topology [8] and social choice [14], rather than on the publication which contains the proofs on equilibrium [11]. I correct the comment's errors and show that the example in the comment is a "mixed economy", while none of my three publications which the comment addresses[11], [8] and [14]-cover mixed economies. In Sections 2 to 6 below I review my results for mixed economies following [16] - cited in the comment as Chichilnisky (1995b) -and show that the comment's example is consistent with my results in [16].

The comment calls attention to my results on the classic Arrow Debreu market without short sales [11] and [16]. I report on these in Sections 2 to 6 . I correct the comment's misunderstandings and errors and show that limited arbitrage is indeed necessary and sufficient for the existence of a competitive equilibrium, with or without short sales, and whether the economy is mixed or not.

*UNESCO Professor of Mathematics and Economics and Director, Program on Information and Resources, Columbia University. Address: 405 Low Library and 618 Mathematics, Columbia University, 117th and Broadway, New York, N.Y. 10027. email gc9@columbia.edu. Comments from Kenneth Arrow, Duncan Foley, Frank Hahn, Geoff Heal, Walt Heller, Ted Groves, Morris Hirsch, Paul Milgrom and Wayne Shafer are gratefully acknowledged.

${ }^{1}[10],[11],[12],[16]$.

${ }^{2}[9],[12],[16],[21]$.

${ }^{3}[7],[12],[14],[16]$. 
The paper is organized as follows. The first part addresses mixed economies following [16], and situates limited arbitrage within the context of a new wider concept, a topological invariant for competitive markets. This topological invariant, denoted $C H$, encodes exact information about the existence of an equilibrium for the economy and its subeconomies, measures social diversity [14] and predicts a failure of 'effective' demand in a sense similar to that of Arrow and Hahn [1]. ${ }^{4}$ A special case is when the invariant is zero: then the market has limited arbitrage. The second part of the paper uses the material in the first part to provide a response to the comment [36].

Sections 2 to 4 summarize my original results on the equivalence of limited arbitrage, the compactness of the Pareto frontier and the existence of an equilibrium given in [10], [11], [12] extending them on the basis of [16] to encompass mixed economies, which are the subject of the comment. Sections 5 and 6 cover social diversity and the failure of effective demand, and Section 7 introduces the topological invariant $C H$. Sections 8 and 9 use the earlier material to provide a detailed response to the comment.

How does limited arbitrage work? Limited arbitrage is strictly weaker than other conditions used to ensure the existence of an equilibrium, which is why it can be necessary and well as sufficient. Other conditions bound the feasible and individually rational trades. Limited arbitrage only bounds the utility levels which are achieved by such trades. The crucial insight is the equivalence of limited arbitrage and the compactness of the Pareto frontier in utility space. ${ }^{5}$ The Pareto frontier is a central concept for resource allocation, and I showed that it controls the existence of an equilibrium, of the core and of satisfactory social choice rules. Limited arbitrage is the non-empty intersection of all market cones. The topological invariant $C H$ is a wider concept: it predicts which cones intersect and which do not. It is based on cohomology and builds on my necessary and sufficient condition for the non-empty intersection of sets of [8].

\section{Definitions}

This section addresses "mixed economies" which is the subject of the comment, on the basis of [16]. This is a class of economies where different traders have different types of preferences, and is larger than the class of economies covered in [11]. The definitions and results provided here are correspondingly more general than those of $[11] .{ }^{6}$ However, I show that the definitions and the results given here are identical to those in [11] when restricted to the smaller domain of "homogenous" economies covered by [11]. This sets up a clear framework for discussing the case of mixed economies and the example provided in the comment, in Sections 8 and 9.

An Arrow Debreu market $\mathrm{E}=\left\{X, \Omega_{h}, u_{h}, h=1, \ldots, H\right\}$ has $H \geq 2$ traders, all with the same trading space $X,{ }^{7} X=R_{+}^{N}$ or $X=R^{N}, N \geq 2$. The results presented here also hold

\footnotetext{
${ }^{4}$ See p. 345 of $[1]$.

${ }^{5}$ The Pareto frontier is the set of undominated and individually rational utility values. This equivalence was established in [18], [22], [10], [12], [11], [16].

${ }^{6}$ The results on limited arbitrage, the Pareto frontier and equilibrium in [11] hold in "homogeneous" economies where either all indifferences have half lines or none do, and where either all traders have indifference surfaces bounded below, or none do, see p. 103, Section 7.0.1, lines 1-5; mixed cases were covered in [16]. These results originated from a theorem in Chichilnisky and Heal [18], a paper which was submitted for publication in February 1984 and was published in February 1993 [22]. Chichilnisky and Heal [22] introduced a non-arbitrage condition $\mathrm{C}$ which is identical to limited arbitrage for preferences without half lines, and proved that $\mathrm{it}$ is sufficient for the compactness of the Pareto frontier (Lemmas 4 and 5) and for the existence of an equilibrium (Theorem 1) with or without half lines, with or without short sales, and in finite or infinite dimensions. [11] and Section 4 below discuss the literature further.

${ }^{7} R_{+}^{N}=\left\{\left(x_{1}, \ldots, x_{N}\right) \in R^{N}: \forall i, x_{i} \geq 0\right\}$.
} 
in infinite dimensions [21]. Traders may have zero endowments of some goods. $\Omega_{h} \in R_{+}^{N}$ denotes trader $h$ 's property rights. When $X=R_{+}^{N}, \Omega_{h} \neq 0 \forall h$ and $\sum_{h} \Omega_{h}=\Omega>>0$.

Assumption 0. Trader $h$ has a preference represented by a continuous, quasiconcave and increasing function $u_{h}: X \rightarrow R^{8}$

The assumptions and the results in this paper are ordinal $;^{9}$ therefore without loss of generality assume that for all $h, u_{h}(0)=0$ and $\sup _{\{x: x \in X\}} u_{h}(x)=\infty$.

Assumption 1. When $X=R_{+}^{N}, \forall r>0$ any boundary ray $\Gamma$ is transversal to $u_{h}^{-1}(r) .{ }^{10}$

Remark 1 Assumption 1 means that either a boundary ray $\Gamma$ does not intersect $u_{h}^{-1}(r)$ or, if it does, the utility $u_{h}$ does not achieve a maximum on $\Gamma .^{11}$

Definition 1 A preference on $R^{N}$ is uniformly non-satiated if it has a utility representation satisfying $\exists K, \varepsilon>0$ such that $K\|x-y\| \geq|u(y)-u(x)| \geq \varepsilon\|x-y\|, \forall x, y \in R^{N}$.

Intuitively, a preference is uniformly non satiated when its rate of increase is uniformly bounded.

The following Assumption 2 restricts the rate of increase of the representing utility, and allows indifferences with or without "flats". Geometrically, this assumption includes two types of preferences: in case (a) every indifference surface contains half lines in every direction, and in case (b) no indifference surface contains half lines in any direction. Formally:

Assumption 2. When $X=R^{N}$, preferences are uniformly non-satiated, and they satisfy one of the following two mutually exclusive conditions: (a) the normalized gradient to any closed set of indifferent vectors define a closed set ${ }^{12}$ or (b) no indifference surface contains half lines.

Remark 2 There is one situation when the case $X=R^{N}$ is formally identical to the case where $X=R_{+}^{N}$ and where all traders have interior endowments: this is when $X=R^{N}$ and all traders' indifference surfaces are bounded below. ${ }^{13}$ In this case short trades are allowed but no trader will trade below the utility level of the initial endowment so short

\footnotetext{
${ }^{8}$ This means that $u(x) \geq u(y)$ if $x \geq y$, and $u(x)>u(y)$ if $x>>y$. Preferences need not be strictly increasing coordinatewise. If $x, y \in R^{N}, x \geq y \Leftrightarrow \forall i x_{i} \geq y_{i}, x>y \Leftrightarrow x \geq y$ and for some $i, x_{i}>y_{i}$, and $x>>y \Leftrightarrow \forall i, x_{i}>y_{i}$.

${ }^{9}$ Namely independent of the utility representations.

${ }^{10} \mathrm{~A}$ boundary ray is an open half line starting from 0 contained in $\partial R_{+}^{N}$. Here we say that a manifold $X \subset R^{N}$ is transversal to another $Y$ at a point $x$ if either $X$ and $Y$ do not intersect, or when they do, neither of their respective tangent fields at $x, T X(x)$ and $T Y(x)$, are contained in the other. Under this assumption, when the indifferences of $u_{h}$ intersect $\partial R_{+}^{N}$ then the function admits a smooth representation on a neighborhood of $R_{+}^{N}$.

${ }^{11}$ Assumption 1 includes strictly convex preferences, preferences with indifferences of positive utility which do not intersect the boundary of the positive orthant such as Cobb Douglas and CES preferences, Leontief preferences $u(x, y)=\min (a x, b y)$, preferences which are indifferent to one or more commodities, such as $u(x, y, z)=\sqrt{x+y}$, preferences with indifference surfaces which contain rays of $\partial R_{+}^{N}$ such as $u(x, y, z)=x$, and preferences defined on a neighborhood of the positive orthant or the whole space, and which are increasing along the boundaries, e.g. $u(x, y, z)=x+y+z$.

${ }^{12}$ I.e. the map $x \rightarrow D u_{h}(x) / \| D u_{h}(x \|$ maps closed sets of an indifference surface into closed sets in the unit sphere. This means that the gradient directions to any indifference surface define a closed set, and corresponds to case (a) in [11], encompassing here preferences whose indifferences are bounded below or not. See Sections 8 and 9 for details.

${ }^{13} \mathrm{~A}$ set $S$ is said to be bounded below when $\exists y \in R^{N}: \forall x \in S, x \geq y$.
} 
trades are bounded below. It is worth observing that uniform non-satiation ensures that if one trader has one indifference surface bounded below, then all this trader's indifference surfaces are bounded below.

Definition $2 A$ mixed economy is one which has more than one type of preferences: some traders have preferences of type (a) and others of type (b), and/or some traders have indifferences bounded below, and others not.

Remark 3 Sections 2-7 of this paper and [16] cover mixed economies; in [11] either all traders had indifferences bounded below or none did, and either all traders were of type $(a)$, or all traders were of type (b), see [11] p. 103, Section 7.0.1., lines 1-5. The definitions and results are however identical to those in [11] for the cases which that paper covered.

Remark 4 It can be shown that if $u$ is concave and non-satiated, then on any two dimensional subspace $S \subset R^{N} \exists \varepsilon>0:|u(y)-u(x)| \geq \varepsilon\|x-y\|, \forall x, y \in S$, see [17].

\subsection{Global and Market Cones}

Two cases, $X=R^{N}$ and $X=R_{+}^{N}$, are considered separately.

- Consider first $X=R^{N}$.

Definition 3 For trader $h$ define the cone of directions $A_{h}\left(\Omega_{h}\right)$ along which utility increases without bound:

$$
A_{h}\left(\Omega_{h}\right)=\left\{x \in X: \forall y \in X, \exists \lambda>0: u_{h}\left(\Omega_{h}+\lambda x\right)>u_{h}(y)\right\}
$$

The rays of this cone intersect all indifference surfaces preferred to $\Omega_{h}$. In case (a) the cone $A_{h}\left(\Omega_{h}\right)$ is open (Proposition 1 of [11]). When augmented by the part of its boundary along which utility never ceases to increase $A_{h}\left(\Omega_{h}\right)$ defines the global cone $G_{h}\left(\Omega_{h}\right)$-both cones are new in the literature: ${ }^{14}$

$$
G_{h}\left(\Omega_{h}\right)=\left\{x \in X \text { and } \sim \exists \arg \max _{\mu \geq 0} u_{h}\left(\Omega_{h}+\mu x\right)\right\}
$$

$G_{h}\left(\Omega_{h}\right)$ treats all convex preferences in a unified way and under Assumption 2 it has a simple structure shown in Proposition 3 of the Appendix: in case (a) when preferences have half lines $G_{h}\left(\Omega_{h}\right)$ it equals $A_{h}\left(\Omega_{h}\right)$, and it equals its closure when indifferences contain no half lines, case (b); $G_{h}\left(\Omega_{h}\right)$ is independent of the initial endowment $\Omega_{h}{ }^{15}$ Therefore this cone is identical to the global cone $G_{h}$ used in [11] to define limited arbitrage, see [11], p. $85,(4)$.

\footnotetext{
${ }^{14}$ The cone $A_{h}\left(\Omega_{h}\right)$ has points in common with Debreu's [23] "asymptotic cone" corresponding to the preferred set of $u_{h}$ at the initial endowment $\Omega_{h}$, in that along any of the rays of $A_{h}\left(\Omega_{h}\right)$ utility increases. Under Assumption 1, its closure $\bar{A}\left(\Omega_{h}\right)$, equals the "recession" cone used by Rockafeller in the 1960's, but not generally: when preferences have "fans" as defined in [16], then the recession cone differs from $\overline{A_{h}}\left(\Omega_{h}\right)$, see [16]. Along the rays in $A_{h}\left(\Omega_{h}\right)$ not only does utility increase forever, but it increases beyond the utility level of any other vector in the space. This condition need not be satisfied by asymptotic cones or by recession cones. For example, for Leontief preferences the recession cone through the endow ment is the closure of the upper contour, which includes the indifference curve itself. By contrast, the cone $A_{h}\left(\Omega_{h}\right)$ is the interior of the upper contour set. Related concepts appeared in Chichilnisky [4], [6], [11] [7] and [13]; otherwise there is no precedent in the literature for global cones $A_{h}\left(\Omega_{h}\right)$ or $\bar{A}_{h}\left(\Omega_{h}\right)$.

${ }^{15}$ The cone $G_{h}\left(\Omega_{h}\right)$ is therefore called "uniform" and denoted also $G_{h}$.
} 
The market cone of trader $h$ is

$$
D_{h}\left(\Omega_{h}\right)=\left\{z \in X: \forall y \in G_{h}\left(\Omega_{h}\right),\langle z, y\rangle>0 .\right\}
$$

$D_{h}$ is the convex cone of prices assigning strictly positive value to all directions in $G_{h}\left(\Omega_{h}\right)$.

- Consider next the case: $X=R_{+}^{N}$.

The market cone of trader $h$ is:

$$
\begin{aligned}
D_{h}^{+}\left(\Omega_{h}\right) & =D_{h}\left(\Omega_{h}\right) \cap S(\mathrm{E}) \text { if } S(\mathrm{E}) \subset \mathrm{N}, \\
& =D_{h}\left(\Omega_{h}\right) \text { otherwise. }
\end{aligned}
$$

where $S(\mathrm{E})$ is the set of supports to rational affordable efficient allocations: ${ }^{16}$

$$
\begin{gathered}
S(\mathrm{E})=\left\{v \in R^{N}: \text { if }\left(x_{1} \ldots x_{H}\right) \in \Upsilon,\left\langle v, x_{h}-\Omega_{h}\right\rangle=0,\right. \\
\text { and } \left.u_{h}\left(z_{h}\right) \geq u_{h}\left(x_{h}\right) \text { then }\left\langle v, z_{h}-x_{h}\right\rangle \geq 0 \forall h\right\},
\end{gathered}
$$

and where $\mathrm{N}$ is the set of prices orthogonal to the endowments. ${ }^{17}$

$$
N=\left\{v \in R_{+}^{N}-\{0\}: \exists h \text { with }\left\langle v, \Omega_{h}\right\rangle=0\right\} .
$$

The market cones $D_{h}^{+}\left(\Omega_{h}\right)$ typically vary with the initial endowments. When $\forall h$ $\Omega_{h} \in R_{++}^{N}, D_{h}^{+}\left(\Omega_{h}\right)=D_{h}\left(\Omega_{h}\right){ }^{18}$

Definition 4 If $X=R^{N}$, E satisfies limited arbitrage when

$$
\text { (LA) } \bigcap_{h=1}^{H} D_{h} \neq \emptyset .
$$

When global cones are independent of the initial endowments, this condition is satisfied simultaneously at every set of endowments. In case (b) $(L A)$ is identical to the no-arbitrage condition $C$ introduced in [18], p. 374, and used there to prove the compactness of the Pareto frontier and the existence of an equilibrium with short sales. This definition of limited arbitrage is also identical to that in [11], p. 89, Section 3.1 in the cases which that paper covers.

\footnotetext{
${ }^{16}$ The expression " $\left(v, x_{h}-\Omega_{h}\right)=0$ " was missing in the definition of $S(E)$ in [11] due to a typographical error, which was corrected in the revised version of [16].

${ }^{17} N$ is empty when $\forall h, \Omega_{h}>>0$.

${ }^{18} R_{++}^{N}$ is the interior of $R_{+}^{N}$. The market cone $D_{h}^{+}$contains $R_{++}^{N}$ when $S(E)$ has a vector assigning strictly pasitive income to all individuals. If some trader has zero income, then this trader must have a boundary endowment.
} 

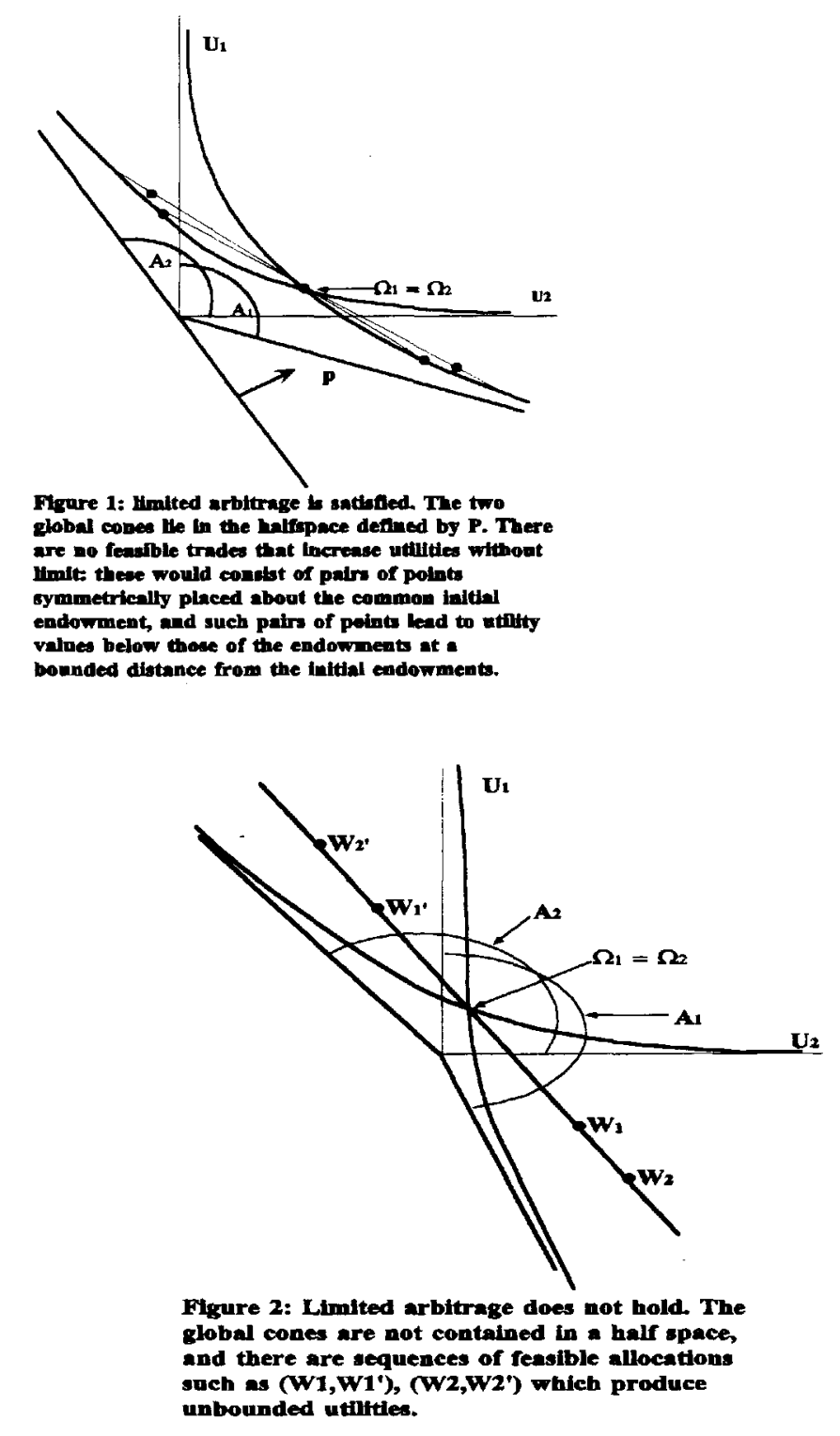

Definition 5 If $X=R_{+}^{N}$, E satisfies limited arbitrage when

$$
\left(L A^{+}\right) \bigcap_{h=1}^{H} D_{h}^{+}\left(\Omega_{h}\right) \neq \emptyset .
$$

This definition is identical to that in [11], (8), p. 91, Section 3.3 .

The set of feasible individually rational trades is $\Upsilon=\left\{\left(x_{1}, \ldots, x_{H}\right) \in X^{H}\right.$ : $\forall h, u_{h}\left(x_{h}\right) \geq u_{h}\left(\Omega_{h}\right)$, and $\left.\sum_{h=1}^{H} x_{h} \leq \Omega\right\}$. The set of feasible individually rational utility allocations is

$$
U(\mathrm{E})=\left\{U=\left(U_{1}, \ldots, U_{H}\right) \in R^{H}: U_{h}=u_{h}\left(x_{h}\right) \geq u_{h}\left(\Omega_{h}\right) \text { where }\left(x_{1}, \ldots, x_{H}\right) \in \Upsilon\right\} .
$$


The Pareto frontier $P(\mathrm{E})$ is the set of undominated vectors in $U(\mathrm{E})$ :

$$
P(\mathrm{E})=\{U \in U(\mathrm{E}): \sim \exists V \in U(\mathrm{E}) \text { with } V>U\} .
$$

A competitive equilibrium is a price vector $p^{*} \in R_{+}^{N}$ and an allocation $\left(x_{1}^{*} \ldots x_{H}^{*}\right) \in$ $\Upsilon$ such that $\sum_{h=1}^{H} x_{h}^{*}-\Omega_{h}=0$ and $x_{h}^{*}$ maximizes $u_{h}$ over the budget set $B_{h}\left(p^{*}\right)=\{x \in$ $\left.X:\left\langle x-\Omega_{h}, p^{*}\right\rangle=0\right\}$.

\subsection{A Financial Interpretation of Limited Arbitrage}

It is useful to show the connection between limited arbitrage and the notion of "noarbitrage" used in finance. The concepts are generally different, but in certain cases they coincide. In financial markets an arbitrage opportunity exists when gains can be made at no cost, or, equivalently, by taking no risks. The simplest illustration of the link between limited arbitrage and no-arbitrage is an economy $E$ where the traders' initial endowments are zero, $\Omega_{h}=0$, and the normalized gradient of a closed set of indifference vectors define a closed set, case (a). Here no-arbitrage at the initial endowments means that there are no trades which could increase the traders' utilities at zero cost: gains from trade must be zero. By contrast, limited arbitrage means that no trader can increase utility beyond a given bound at zero cost; as seen below in 3.1, gains from trade are bounded. In brief: in this example no-arbitrage requires that there should be no gains from trade at zero cost while limited arbitrage requires that there should be only bounded or limited gains from trade at zero cost.

In case (b) limited arbitrage has a stricter meaning: it implies that traders will not engage in unboundedly large transactions; this is identical to the no-arbitrage condition $C$ introduced in [18], p. 374 .

When the traders' utilities are linear functions, the two concepts coincide: there is limited arbitrage if and only if there is no-arbitrage as defined in finance. In brief: in linear economies, limited arbitrage "collapses" into no-arbitrage.

\section{Limited Arbitrage and the Compactness of the Pareto Frontier}

The Pareto frontier $P(\mathrm{E})$ is the set of feasible, individually rational and undominated utility allocations. The compactness (and nonemptiness) of the Pareto frontier is a crucial step in establishing the existence of a competitive equilibrium.

It is useful to clarify the difference between limited arbitrage and conditions used elsewhere. ${ }^{19}$ Other conditions used in the literature require a compact set of feasible individually rational allocations $\Upsilon{ }^{20}$ For example, the no-arbitrage condition $\mathrm{C}$ of [22] ensures

\footnotetext{
${ }^{19}$ Case (b), when indifferences contain no half lines, is particularly simple: under limited arbitrage feasible and rational trades always define a compact set, exactly as in economies without short sales.

${ }^{20}$ Without short sales the set $\Upsilon$ is always oompact. In economies with short sales, Chichilnisky and Heal [18], [22] introduced the "no-arbitrage condition $\mathrm{C}$ ", and similar conditions were used in Werner [41], Nielsen [38], Koutsougeras [35] (and several others), the latter three based on recession cones. Any no-arbitrage condition based on recession cones amounts to a compact set of feasible and desirable allocations $\Upsilon$ in commodity space, see e.g. Lemma 2 of Chichilnisky [11] and Lemma 10.1, Section 10, p. 20 of Koutsougeras [35]. Werner's sufficient condition for existence is somewhat weaker because he removes some constant directions from the recession cone, but like all the others it is strictly stronger than limited arbitrage. He requires $S=\cap S_{i} \neq \emptyset$, (see Theorem 1 on p. 1410 of [41]) where $S_{i}=\{p:\langle p, x\rangle>0$ for all $\left.x \in W_{i}\right\}$ (bottom of p. 1409), and where $W_{i}$ is the recession cones minus the directions along which
} 
a compact set of individually rational and feasible trades $\Upsilon$, and thus the compactness of $P(\mathrm{E}) .^{21}$ By contrast, limited arbitrage ensures a compact utility possibility set, without requiring the compactness of the underlying set of trades $\Upsilon$ see [7], [10], [11], [12], [14], [13], [16], and [20]. It has been established within humogeneous economies that limited arbitrage is necessary and sufficient for a compact utility possibility set $U(\mathrm{E})$, and hence the compactness of its boundary $P(E),{ }^{22}$ for details see [10], [11], [19], [22]. The following Theorem extends this result to mixed economies following [16].

Theorem 1 Consider an economy $\mathrm{E}$ as defined in Section 1. Then limited arbitrage is necessary and sufficient for the compactness of the set of feasible and individually rational utility allocations $U(\mathrm{E})$, and therefore for the compactness and non-emptiness of the Pareto frontier $P(E)$. Feasible allocations and undominated utility allocations may however define unbounded sets.

Proof. See the Appendix.

Proposition 1 When $X=R^{N}$, limited arbitrage implies that the Pareto frontier $P(E) \subset$ $R_{+}^{N}$ is homeomorphic to a simplex. ${ }^{23}$

Proof. This follows from Theorem 1 and the convexity of preferences, cf. Arrow and Hahn [1]

\subsection{Interpretation of Limited Arbitrage as Bounded Gains From Trade when $X=R^{N}$}

Limited arbitrage has a simple interpretation in terms of gains from trade when $X=R^{N}$. Gains from trade are defined as follows:

$$
\mathrm{G}(\mathrm{E})=\sup _{\left(x_{1}, \ldots x_{H}\right) \in \Upsilon} \sum_{h=1}^{H} u_{h}\left(x_{h}\right)-u_{h}\left(\Omega_{h}\right) ;
$$

$\Upsilon$ was defined above as the set of feasible and individually rational allocations.

Proposition 2 The economy $\mathrm{E}$ satisfies limited arbitrage if and only it has bounded gains from trade which are attainable, i.e. $\exists x^{*} \in \Upsilon$ :

$$
\mathrm{G}(\mathrm{E})=\sum_{h=1}^{H} u_{h}\left(x_{h}^{*}\right)-u_{h}\left(\Omega_{h}\right)<\infty .
$$

the utility is constant, see 1408 , p. 6, last two lines. Observe that $W_{i}$ contains many directions which are not included in $G_{h}$ such as those directions along which the preference is eventually constant without being constant. Such directions are not part of the global cone $G_{h}$ defined here and in [11]. Therefore the cone $W_{i}$ is strictly larger than the global cone $G_{h}$, and the condition $S \neq \emptyset$ is strictly stronger than limited arbitrage as defined here and in [11]. Werner's condition is therefore not necessary for existence of an equilibrium unless indifferences contain no half lines, see also Proposition 2, (ii), p. 1410 of [41]. In this latter case, Werner's condition bounds the set of all feasible individually rational allocations $\Upsilon$ as do all other non-arbitrage conditions except for limited arbitrage.

${ }^{21}$ Lemmas 4 and 5 of [18] prove the compactness of $P(E)$ using its "no-arbitrage condition $C$ " which is identical to limited arbitrage when indifferences have no half lines, see $[18]$ p. 374, [11] and [16].

${ }^{22}$ Lemma 2 of $[11]$ established that limited arbitrage is sufficient for compactness of $P(\mathrm{E})$ and Theorem 1 of [11], by the second welfare theorem, proved that limited arbitrage is necessary for compactness of $P(E)$, because it is necessary for the existence of an equilibrium.

${ }^{23}$ A topological space $X$ is homeomorphic to another $Y$ when there exists a on-to-one onto map $f$ : $X \rightarrow Y$ which is continuous and has a continuous inverse. 
Proof. Proposition 3 in the Appendix shows that under the conditions global cones are independent of the initial endowments, so we may assume without loss of generality that $\Omega_{h}=0$ for all $h$. Sufficiency first. The sum $\sum_{h=1}^{H} u_{h}$ defines a continuous function of $\left(u_{1}, \ldots, u_{H}\right) \in R_{+}^{H}$; this continuous function always attains a maximum when the vector $u_{1}, \ldots, u_{h}$ varies over the set $U(\mathrm{E}) \subset R_{+}^{H}$, which is compact by Theorem 1 when limited arbitrage is satisfied. The converse is immediate from the proof of necessity in Theorem 1 , which establishes that when limited arbitrage fails, there exist no undominated individually rational utility allocations, i.e. $P(\mathrm{E})$ is empty.

Corollary 1 In case (a) the economy has limited arbitrage if and only if it has bounded gains from trade, i.e. ${ }^{24}$

$$
\mathrm{G}(\mathrm{E})<\infty
$$

Proof. Recall that in case (a), $G_{h}=A_{h}$ by Proposition 3 in the Appendix. In this case limited arbitrage is also necessary for bounded gains from trade.

\section{Competitive Equilibrium and Limited Arbitrage}

It was proved in [11] that limited arbitrage is simultaneously necessary and sufficient for existence of a competitive equilibrium in economies with or without short sales. ${ }^{25}$ This result was established in [11] for economies where preferences were homogeneous: either all preferences are of type (a) or all of type (b), and either all have indifferences bounded below or none do. Below I show that this is true for mixed economies, where some preferences may be of type (a) and others of type (b), where some indifferences are bounded below and others are not. This follows [16]. This result includes the classic economy of Arrow and Debreu without short sales, which was neglected previously in the literature on no-arbitrage. The equivalence between limited arbitrage and equilibrium extends to economies with infinitely many markets, see [21].

Recall that limited arbitrage implies a compact set of feasible and individually rational utility allocations $U(\mathrm{E})$, without requiring that the underlying trades $\Upsilon$ be compact. Therefore under limited arbitrage the set of equilibria, the set of undominated utility allocations and the core may all be unbounded when $X=R^{N}$.

Theorem 2 Consider an economy $\mathrm{E}=\left\{X, u_{h}, \Omega_{h}, h=1, \ldots, H\right\}$, where $H \geq 2$, with $X=R^{N}$ or $X=R_{+}^{N}$ and $N \geq 1$. Then the following two properties are equivalent:

\footnotetext{
${ }^{24}$ In the following equation " $\infty$ " must be replaced by $\sup _{\{x: x \in X)}\left(\sum_{h=1}^{H} u_{h}(x)-u_{h}\left(\Omega_{h}\right)\right)$ when $\forall h$, $\sup _{x \in X} u_{h}(x)<\infty$.

${ }^{25}$ Chichilnisky and Heal [18], Hart [28] Hammond [27] and Werner [41] among others, have defined various no-arbitrage conditions which they prove, under certain conditions on preferences, to be sufficient for existence of equilibrium. Hart and Hammond study asset market models which are incomplete economies because they lack forward markets, and therefore have typically inefficient equilibria. None of these noarbitrage conditions is generally necessary for existence. For the special case (b), within economies with short sales (which exclude the classic Arrow Debreu's market), and where recession cones are assumed to be uniform, Werner [41] remarks correctly (p. 1410, last para.) that another related condition (p. 1410 , line -3 ) is necessary for existence, without however providing a complete proof of the equivalence between the condition which is necessary and that which is sufficient. The two conditions in [41] are defined on different sets of cones: the sufficient condition is defined on cones $S_{i}$ (p. 1410, line -14) while the necessary condition is defined on other cones, $D_{i}$ (p. 1410, -3). The equivalence between the two cones depends on properties of yet another family of cones $W_{i}$ (see p. 1410, lines 13-4). The definition of $W_{i}$ on page 1408 , line -15 shows that $W_{i}$ is different from the recession cone $R_{i}$, (which are uniform by assumption) and therefore the cone $W_{i}$ need not be uniform even when the recession cones are, as needed in Werner's Proposition 2.
} 
(i) The economy $\mathrm{E}$ has limited arbitrage

(ii) The economy $\mathrm{E}$ has a competitive equilibrium.

Proof. See the Appendix.

Remark 5 When $X=R^{N}$ the set of competitive equilibria need not be bounded.

\subsection{Subeconomies with Competitive Equilibria}

As shown in [11] the condition of limited arbitrage need not be tested on all traders simultaneously: in the case of $R^{N}$, it needs only be satisfied on subeconomies with no more traders than the number of commodities in the economy, $N$, plus one.

Definition 6 A $k$-trader sub-economy of $E$ is an economy $F$ consisting of a subset of $k \leq$ $H$ traders in $\mathrm{E}$, each with the endowments and preferences as in $\mathrm{E}: \mathrm{F}=\left\{X, u_{h}, \Omega_{h}, h \in\right.$ $J \subset\{1, \ldots, H\}$, cardinality $(J)=k \leq H\}$.

Theorem 3 The following four properties of an economy $\mathrm{E}$ with trading space $R^{N}$ are equivalent:

(i) E has a competitive equilibrium

(ii) Every sub economy of $\mathrm{E}$ with at most $N+1$ traders has a competitive equilibrium

(iii) E has limited arbitrage

(iv) E has limited arbitrage for any subset of traders with no more that $N+1$ members.

Proof. Theorem 2 implies (i) $\Leftrightarrow($ iii) and (ii) $\Leftrightarrow($ iv). That (iii) $\Leftrightarrow$ (iv) follows from Helly's theorem, which is a corollary in Chichilnisky [8]: Consider a family $\left\{U_{i}\right\}_{i=1 \ldots H}$ of convex sets in $R^{N}, H, N \geq 1$. Then

$$
\bigcap_{i=1}^{H} U_{i} \neq \emptyset \text { if and only if } \bigcap_{j \in J} U_{i} \neq \emptyset
$$

for any subset of indices $J \subset\{1 \ldots H\}$ having at most $N+1$ elements.

In particular, an economy $E$ satisfies limited arbitrage, if and only if it satisfies limited arbitrage for any subset of $k=N+1$ traders, where $N$ is the number of commodities in the economy $\mathrm{E}$.

\section{A failure of 'effective' demand when $X=R_{+}^{N}$.}

In economies without short sales $X=R_{+}^{N}$ a compensated equilibrium or quasiequilibrium always exists, Arrow and Hahn [1] and Negishi [37]. However, when limited arbitrage fails, quasiequilibria are ill-behaved; I show below that every quasiequilibrium has a failure of 'effective' demand, in the sense of Arrow and Hahn [1], p. 345.

Definition 7 A compensated equilibrium or quasiequilibrium is a price $p^{*}$ and an allocation $x^{*}$ at which every trader minimizes the cost of achieving the given utility level, and $\sum_{h=1}^{H}\left(x_{h}^{*}-\Omega_{h}\right)=0$. 
Although a quasiequilibrium allocation $x^{*}$ satisfies $\sum_{h=1}^{H}\left(x_{h}^{*}-\Omega_{h}\right)=0, x^{*}$ can exhibit 'excess demand', if demand is computed from utility maximization under a budget constraint. This is because at the quasiequilibrium allocation traders minimize costs, but may not maximize utility. If at a quasiequilibrium allocation every trader maximizes utility, then the quasiequilibrium is also a competitive equilibrium.

Definition 8 An allocation and a price exhibit a failure of effective demand in the sense of [1] when in some markets there exists excess demand (computed from utility maximization subject to a budget constraint), but the value of this excess demand is zero.

Theorem 4 When $X=R_{+}^{N}$ a failure of limited arbitrage implies that there is a failure of effective demand: every quasiequilibrium has excess demand, but the market value of this demand is zero.

Proof. A quasi equilibrium price $p^{*}=\left(p_{1}^{*}, \ldots, p_{N}^{*}\right) \in R_{+}^{N}$ always exists because $X=$ $R_{+}^{N}$. As seen in Theorem 2 if limited arbitrage fails there is no competitive equilibrium. This implies that at any quasiequilibrium some trader has zero income (for otherwise a quasiequilibrium is always a competitive equilibrium). Therefore for at least one market $i, p_{i}^{*}=0$, and the value of excess demand in the $i-t h$ market is therefore zero. For some trader $h, p^{*} \notin D_{h}^{+}$(since otherwise the quasiequilibrium would be a competitive equilibrium) so that there is no maximum for $u_{h}$ on the budget set defined by $p^{*}$. Therefore there is excess demand at $p^{*}$. in the $i-t h$ market, but the value of excess demand in this market is zero.

\section{Social Diversity and Limited Arbitrage}

If the economy does not have limited arbitrage, it is called socially diverse:

Definition 9 When $X=R^{N}$, the economy is socially diverse if $\bigcap_{h=1}^{H} D_{h}=\emptyset$

This concept is independent of the units of measurement or choice of numeraire.

Social diversity admits as many different "shades" as traders:

Definition 10 The economy $\mathrm{E}$ has index of diversity $I(\mathrm{E})=H-K$ if $K+1$ is the smallest number such that $\exists J \subset\{1 \ldots H\}$ with cardinality of $J=K+1$, and $\bigcap_{h \in J} D_{h}=\emptyset$. The index $I(\mathrm{E})$ ranges between 0 and $H-1$ : the larger the index, the larger the social diversity. The index is smallest $(=0)$ when all the market cones intersect: then all social diversity disappears, and the economy has limited arbitrage.

Theorem 5 The index of social diversity is $I(E)$ if and only if $H-I(E)$ is the maximum number of traders for which every subeconomy has a competitive equilibrium.

Proof. This is immediate from Theorem 3.

\section{A topological invariant for competitive markets}

This section introduces a new and useful concept, a topological invariant for competitive markets. This is an algebraic object which describes important properties of the market-its social diversity and the existence of an equilibrium for it or for its 
subeconomies-in a way that is robust, namely independent of the units of measurement. The topological invariant $C H$ introduced here contains exact information about the resource allocation properties of the economy, as shown below. Furthermore it is computable by simple algorithms from the initial data of the problem: its endowments and preferences.

Important properties of an economy $E$ with short sales can be described in terms of the properties of a family of cohomology rings denoted $C H(\mathrm{E})$. The cohomology rings ${ }^{26}$ of a space $Y$ contain information about the topological structure of $Y$, namely those properties of the space which remain invariant when the space is deformed as if it was made of rubber. For formal definitions of the algebraic topology concepts used here a standard textbook is Spanier [40].

The following concept, called a nerve, defines a combinatorial object, called a simplicial complex, from any family of sets. Starting from any arbitrary family of sets in $R^{N}$, one defines from it a triangulated set, obtained by "pasting up" simplices in a well ordered fashion, see [40], called a simplicial complex. The simplicial complex created from the family of sets is usually called the "nerve of the family of sets". The procedure for transforming any family of sets into a simplicial complex is as follows: each set is represented by a point, which is a vertex in the triangulated space; the intersection of any two sets in the family is represented as a one-simplex, the intersection of any three sets as a 2-simplex, etc. It is simple to see that putting all this together one obtains a simplicial complex [40]. Formally:

Definition 11 The nerve of a family of subsets $\left\{V_{i}\right\}_{i=1, \ldots, L}$, in $R^{M}$, denoted

$$
\text { nerve }\left\{V_{i}\right\}_{i=1, \ldots, L}
$$

is a simplicial complex defined as follows: each subfamily of $k+1$ sets in $\left\{V_{i}\right\}_{i=1, \ldots, L}$ with non-empty intersection is a $k$-simplex of the nerve $\left\{V_{i}\right\}_{i=1, \ldots, L}$.

Now that the nerve of a family of sets is defined, one looks at the global topology of this simplicial complex, in words, how many "holes" it has, and of what type. This is measured by the cohomology rings of the space, which are groups with an additional operation, which measure precisely the number and types of holes which the space has. The cohomology rings are computable by standard algorithms once the market cones of the economy are known. See Spanier for definitions [40]. Now we are ready to define our topological invariant.

A subfamily of the family of sets $\left\{D_{h}\right\}_{h=1, \ldots, H}$ is a family consisting of some of the sets in $\left\{D_{h}\right\}_{h=1, \ldots, H}$, and is indicated $\left\{D_{h}\right\}_{h \in Q}$ where $Q \subset\{1, \ldots, H\}$. The topological invariant $C H(\mathrm{E})$ of the economy $\mathrm{E}$ with $X=R^{N}$ is the family of reduced cohomology rings ${ }^{27}$ of the simplicial complexes defined by all subfamilies $\left\{D_{h}\right\}_{h \in Q}$ of the family $\left\{D_{h}\right\}_{h=1,2 \ldots H}$, i.e. the cohomology rings of nerve $\left\{D_{h}\right\}_{h \in Q}$ for every $Q \subset\{1, \ldots, H\}$ :

$$
C H(\mathrm{E})=\left\{H^{*}\left(\text { nerve }\left\{D_{h}\right\}_{h \in Q}, \forall Q \subset\{1, \ldots, H\}\right\}\right.
$$

In the following result I consider continuous deformations of the economy which preserve all the Assumptions in Section 2. Any such deformation will preserve our topological

\footnotetext{
${ }^{26} \mathrm{~A}$ ring is a set $Q$ endowed with two operations, denoted + and $\times$; the operation + must define a group structure for $Q$ (every element has an inverse under + ) and the operation $\times$ defines a semi group structure for $Q$; both operations together satisfy a distributive relation. A typical example of a ring is the set of the integers, another the rational numbers, both with addition and multiplication.

${ }^{27}$ With integer ooefficients.
} 
invariant. The following result shows how much information is encoded in $C H(\mathrm{E})$. It shows that the existence of an equilibrium for the economy and its subeconomies are purely topological properties, and they are predicted exactly by the number and the type of 'holes' in the nerve of the family of market cones of the economy, i.e. by the cohomology rings of this nerve. This result depends on a new theorem in Chichilnisky [8] which established that a family of finitely many convex sets has non empty intersection if and only if each subfamily has a contractible union:

Theorem 6 The economy $\mathrm{E}$ with $H$ traders has limited arbitrage, and therefore a competitive equilibrium if and only if:

$$
\begin{gathered}
C H(\mathrm{E})=0 \\
\text { i.e. } \forall Q \subset\{1, \ldots, H\}, \mathrm{H}^{*}\left(\text { nerve }\left\{D_{h}\right\}_{h \in Q}\right)=0
\end{gathered}
$$

Furthermore, the economy $\mathrm{E}$ has social diversity index $I(\mathrm{E})$ if and only if $I(E)=H-K$, where $K$ satisfies the following conditions: (i) for every set $Q \subset\{1, \ldots, H\}$ of cardinality at most $K$

$$
\mathrm{H}^{*}\left(\text { nerve }\left\{D_{h}\right\}_{h \in Q}\right)=0,
$$

and there exists $T \subset\{1, \ldots, H\}$ with cardinality $T=K+1$ and

$$
H^{*}\left(\text { nerve }\left\{D_{h}\right\}_{h \in T}\right) \neq 0 .
$$

Proof. This follows from Theorem 5 and Corollary 2 to Theorem 6, p. 200 of Chichilnisky [8], which proves that an acyclic family of sets has non empty intersection if and only if every subfamily has acyclic union, ${ }^{28}$ see [8] for details.

\section{Response and Examples}

This section and Section 9 give more detailed responses to a comment [36] which focusses on the announcements contained in my publications [8] and [14]; the comment also mentions my original results in [11]. Sections 8 and 9 are independent of the rest of the paper, and can be skipped by the informed reader without lass.

This section discusses several examples and points out errors in the comment [36] which, when corrected, invalidate its conclusions. Some of the errors appear repeated in difference contexts within [36], requiring the response to address them, correspondingly, more than once. In addition, example 3 below shows that limited arbitrage is strictly weaker than resource relatedness [1].

The comment [36] consists of a simple example of a "mixed economy" with two traders. One trader's indifferences are bounded below, and the second trader's are not; one trader has indifferences with half lines and the other does not. This is a type of economy which I never claimed to cover in my work which the comment addresses. The authors erred by focussing on two papers which cover other areas, algebraic topology [8] and social choice [14] ${ }^{29}$ rather than on the publication which contains the proofs on equilibrium [11]. Theorem 1 of [11] does not explicitly mention whether mixed cases are covered, but it is clear to anyone who reads my proofs in [11] that they were not intended to apply

\footnotetext{
${ }^{28}$ This result applies also to more general families.

${ }^{29}$ These two papers [14], [8] prove results in other areas-algebraic topology and social choice respectively-and only mention equilibrium in brief announcements without details or proofs. For proofs the reader was referred elsewhere [11].
} 
to the mixed case.$^{30}$ In addition, the authors of the comment misread my definition of global cones. They say that their example satisfies limited arbitrage, but it does not. This error has already been pointed out in [29], and by itself invalidates the conclusions of the comment.

The mixed case was covered in another paper [16], which the three authors cite in their comment as Chichilnisky (1995b). [16] extends the definition of the global cone in [11] to encompass mixed economies; both definitions are identical in the economies which both papers cover. My results for mixed economies in [16] are consistent with the comment's example, but the three authors fail to mention this fact. In sum: the comment contains only special cases of my results. My Theorem 1 in [11] proves that limited arbitrage is necessary and sufficient for the existence of a competitive equilibrium in homogeneous economies, a result which holds true exactly as stated and proved. ${ }^{31}$

The literature on no arbitrage had neglected the classical economy of Arrow and Debreu without short sales. My results on this subject are highlighted in the comment. It discuses two figures in [11] (4B and 7). The authors have misread my conditions and my results and state, incorrectly, that these figures contradict my results. They do not, and it is simple enough to correct the comment's error. As shown below, these figures are simple illustrations for Theorem 1 of [11].

\subsection{Mixed Economies}

Example 1. My results in [11] dealt only with homogenous economies: the statement of Theorem 1 in [11] does not mention whether mixed economies were included, but it is clear to anyone who read my proofs that I covered only homogeneous economies, see p. 103, Section 7.0.1., lines 1-5 of [11]. By contrast, the comment's example is not a homogeneous economy, as shown below. Therefore, the comment's example is not a counterexample to the results in the publications which it addresses, all of which pertain the proofs in [11]. This is easy to check: [36] consists of one simple example of an economy with short sales, two traders and two goods. Trader's one utility is $u_{1}\left(x_{1}, x_{2}\right)=$ $x_{1}+x_{2}+2-\sqrt{\left(x_{1}-x_{2}\right)^{2}+4}$, and trader two's utility is $u_{2}\left(x_{1}, x_{2}\right)=x_{1}{ }^{32}$ This is a "mixed economy," because trader one's indifferences are bounded below, while trader two's are not, and trader one's indifferences have no half lines while trader two's indifferences have half lines. As stated in Lemma 2 [11] Section 7.0.1, p. 103, lines 1-5, I considered the two cases separately: homogeneous economies where either all traders have indifferences bounded below, which is not really new, or the new case where all indifference surfaces are unbounded below. ${ }^{33}$

Therefore the comment's example is irrelevant to the publications which it addresses. It deals with a "mixed economy", a type of economy which was covered in my paper [16],

\footnotetext{
${ }^{30}$ See e.g. Lemma 2 of [11], p. 103, Section 7.0.1, lines 1-5.

${ }^{31}$ When all traders have indifferences bounded below, or when none do.

${ }^{32}$ See [36] Section 3, Figures 1(a, 1(b), 2(a) and 2(b), and subsection 3.2.

${ }^{33}$ I quote from [11], p. 103, Section 7.0.1., lines 1-5: "When all indifference surfaces of the preferences are bounded below the proof is identical to the case where $X=R_{+}^{N}$ a case where the result is known (cf. Arrow and Hahn [1]). Therefore we need only consider the case where $X=R^{N}$ and where the indifference surfaces of the traders are not all bounded below", lines 1-5, p. 103, Section 7.0.1. of [11]. Observe that by Assumption 2 on page 84 of [11], or Assumption 2 above, if one indifference surface of a trader is bounded below, then all of his/her indifference surfaces are bounded below. Therefore the two exclusive cases considered in [11] are either: all indifference surfaces are bounded below, or none are. It is clear that the example in Section 3 of the comment does not satisfy my conditions because it mixes two types of preferences.
} 
which is cited in the comment as Chichilnisky (1995b). It is therefore of interest to point out that the comment's example is consistent with the results on mixed economies of [16].

In addition to misunderstanding my hypothesis, the three authors made another erros. They misread my definitions and erred in the definiticn of global cones and limited arbitrage, as shown below. This point was already made in [29] - see the quote below-and is explained in what follows. Because their example does not satisfy limited arbitrage, it does not have a competitive equilibrium. Thus their example confirms Theorem 2 for mixed economies in [16], reported also in this paper, Theorem 2 above.

The details are as follows. On the last line of Section 3.1 of [36] the authors state "Thus limited arbitrage is satisfied." However, the three authors made an error: this economy does not have limited arbitrage. By the definition of $G_{h}$ in (1) (see [16] and also [11], p. 85 (4)), the first trader in the example of [36] Section 3 has as global cone $G_{1}=\left\{x_{1}, x_{2}: x_{1} \geq 0\right.$ and $\left.x_{2} \geq 0\right\}$, i.e. the closed positive orthant, because this trader's indifferences contain no half lines (this is case (b) of [11], p. 85 (4)). However the three authors got this wrong, stating that my global cone $G_{h}$ is the set they denote $A_{1}$ in the statement of their Theorem $3[36]$. As already seen, however, my definition of global cones given in [16] and in [11] p. 85, (4) states clearly that when preferences contain no half lines (case (b)) the global cone $G_{h}$ is the closure of the set $A_{h}$, i.e. it is the set they denote $I_{1}$ in Theorem 3 of [36]. The three authors confused $I_{1}$ with $A_{1}$. This is an error which invalidates the rest of the statements in the comment.

The second trader has half-lines in the indifferences (case (a)), and by definition of $G_{h}$ in (1), $G_{h}$ is open, indeed it is the open half space defined by $G_{2}=\left\{x_{1}, x_{2} \in R^{2}: x_{1}>0\right\}$, as the authors themselves point out in Theorem 3 of Section 3.2.

It is now immediate to observe that, contrary to what the comment states, limited arbitrage is not satisfied in their example in Section 3, Theorem 3 of [36]. The market cones are respectively the open pasitive orthant $D_{1}=\left\{\left(x_{1}, x_{2}\right): x_{1}>0\right.$ and $\left.x_{2}>0\right\}$ and the vertical line $D_{2}=\left\{\left(x_{1}, x_{2}\right)=x_{1}=0\right\}$. Since $D_{1} \cap D_{2}=\emptyset$, limited arbitrage is not satisfied. This economy has no equilibrium, as the comment states. Since there is no equilibrium and limited arbitrage fails, this example is consistent with my results in Theorem 2 of [16], which are reported also in this paper. It appears that the authors have misread my papers.

This point was clearly stated in a letter dated January 10, 1996 from Heller, Groves. Hirsch. Shafer and others [29] addressed to Monteiro, Page and Wooders:

"You recently circulated two papers which claim to find counterexamples to recently published papers by Graciela Chichilnisky. Her papers [11], [8] and [14] contain two very important and original results on arbitrage, the Pareto frontier and equilibrium.... Of the three papers on which you comment, this [11] contains the most recent and complete statements...However, you have misread a definition, a proposition and a lemma. You have also omitted some of Chichilnisky's assumptions in obtaining your "counterexample."....The example in Monteiro Page and Wooders' second paper is built on "mixed economies" where different agents have different types of preferences (bounded or unbounded below, half lines and no half lines). Monteiro, Page and Wooders take agent 1 to fall in case (a) whereas a natural interpretation is that agent 1 falls in the explicit "no-half lines" case (b). Thus agent 1's global cone is closed, limited arbitrage fails and there is no inconsistency with Chichilnisky's results."

A subsequent letter [30] from W. Heller and M. Hirsch addressed to F. Page and M. 
Wooders dated February 29, 1996, states:

"It is misleading to say that you have counterexamples to theorems in Chichilnisky's papers in $B A M S$ and $A E R$. The theorems in question were only announced there, not proved. If you want to say that the theorems were stated too tersely, that is one thing. To say that they are wrong is quite another and we do not agree with you on that point"

Both letters [29] and [30] are available from the author upon request.

\subsection{Markets without short sales}

Example 2. The comment [36] refers briefly to my results on the classic Arrow Debreu economy without short sales in its Section 5, last paragraph. The three authors claim that my example in Figure $4 \mathrm{~B}$ of [11], p. 88, has a competitive equilibrium but does not satisfy limited arbitrage. This is incorrect, because this example does not have a competitive equilibrium. This is in accordance with Theorem 1 of [11] and Theorem 2 above and in [16]. Their example thus confirms my results.

Figure 4B in [11] is a two person economy with trading space $X=R_{+}^{2}$. Trader one owns $\Omega_{1}=\left(0, x_{2}\right)$, a vector consisting only of second good $^{34}$ and trader two owns a vector $\Omega_{2}$ which is in the interior of the positive orthant. ${ }^{35}$ Trader one has a preference which is strictly increasing in both goods. ${ }^{36}$ For trader one the global cone is $G_{1}\left(\Omega_{1}\right)=R_{+}^{2}=$ $\left\{\left(x_{1}, x_{2}\right): x_{1} \geq 0\right.$ and $\left.x_{2} \geq 0\right\}$ i.e. the whole pasitive orthant ${ }^{37} R_{+}^{2}$ and by definition $D_{1}=\left\{\left(x_{1}, x_{2}\right): x_{1}>0\right.$ and $\left.x_{2}>0\right\}$. Trader two is indifferent in the second good as shown in Figure $4 \mathrm{~B}$ of [11]. Furthermore since this trader has an interior endowment ${ }^{38} \Omega_{2}$ the only price in the $\operatorname{set}^{39} S(E)$ is the vector $v=(1,0)$ as indicated in Figure $4 \mathrm{~B} .{ }^{40}$ Since $\left\langle v, \Omega_{1}\right\rangle=0$, by definition $S(E) \subset N$. Therefore by definition $D_{1}^{+}=D_{1} \cap S(E)=\emptyset .{ }^{41}$ It follows that limited arbitrage is not satisfied in Figure $4 \mathrm{~B}$, because $D_{1}^{+}=\emptyset \Rightarrow D_{1}^{+} \cap D_{2}^{+}=$ $\emptyset$. By Theorem 2 above (see also Theorem 2 of [16]) this economy has no competitive equilibrium. This is easy to verify. The only possible supporting price is $v$, at which excess demand of trader one is not well defined. The comment went wrong by stating that in my example "one trader likes only one good and is endowed with the other good" see last paragraph of Section 5 of [36]. Neither of my two traders has the characteristic described by the comment: trader one only owns the second good and prefers both, and trader two owns both goods and prefers only the first. As detailed above, this example fails to have limited arbitrage since $D_{1}^{+} \cap D_{2}^{+}=\emptyset$. Since there is no equilibrium and limited arbitrage fails, this example confirms my Theorem 2 above [16], and Theorem 1 of [11].

\subsubsection{Limited arbitrage is strictly weaker than resource relatedness}

Example 3. This example is Figure $4 \mathrm{~A}$ in [11], p. 89. It shows that limited arbitrage is strictly weaker than resource relatedness - even though it is still sufficient for the existence

\footnotetext{
${ }^{34}$ See $[11]$ page 89 , lines 3 and $10-11$.

${ }^{35}$ See lines $1-2$ and $10-11$, p. 89 of $[11]$.

${ }^{36}$ There is an obvious switch in the indices 1 and 2 here but in any case the argument is clear.

${ }^{37}$ As stated in lines $12-15$ p. 89 of $[11]$.

${ }^{38}$ As stated in lines $1-2$ and $10-11$ p. 89 of $[11]$.

${ }^{39}$ As mentioned above in $\mathrm{ft}$. , the definition of $S(E)$ missed the expression " $\left(v, x_{h}-\Omega_{h}\right)=0$ " in [11], but this appeared correctly in the revised version of [16] which the three authors of [36] have.

${ }^{40}$ And as stated in lines $13-15$ p. 89 of [11].

${ }^{41}$ As stated in page 89 , line 13 and in my Figure $4 \mathrm{~B}$ of [11].
} 
of an Arrow Debreu equilibrium. Figure 4A depicts a two-trader two-good economy without short sales, where one trader only owns good two, $\Omega_{1}=\left(0, x_{1}\right)$ and the other owns both $\Omega_{2}=\left(y_{1}, y_{2}\right), y_{1}>0$ and $y_{2}>0$. Both traders are indifferent about good two, having the same utility function $u(z, w)=z$. This exanple has a competitive equilibrium: it is the initial endowment with prices $(1,0)$; the economy also satisfies limited arbitrage as Theorem 1 of [11] predicts. However, this economy does not satisfy resource relatedness, because trader one's endowment has no value for trader two. This shows that limited arbitrage is strictly weaker than resource relatedness. The details are as follows. As shown above, the only vector in $S(E)$ is $v=(1,0)$, because $\Omega_{2}>>0$ and $u(z, w)=z$. Furthermore $\left\langle v, \Omega_{1}\right\rangle=0$, so that $S(E) \subset N$. Here $G_{1}\left(\Omega_{1}\right)=G_{2}\left(\Omega_{2}\right)=\left\{\left(x_{1}, x_{2}\right): x_{1}>0\right.$ and $\left.x_{2} \geq 0\right\}$ and $D_{1}=D_{2}=\left\{\left(x_{1}, x_{2}\right): x_{1}>0\right.$ and $\left.x_{2} \geq 0\right\}$, so that $D_{2}^{+}=D_{1}^{+}=$ $D_{1} \cap S(E)=\{v\} \neq \emptyset$, and $D_{1}^{+} \cap D_{2}^{+}=\{v\} \neq \emptyset$. Therefore limited arbitrage is satisfied and, as proved in Theorem 1 of [11] and Theorem 2 above [16], a competitive equilibrium exists. Yet resource relatedness is not satisfied.

Example 4. The final example refers to Figure 7 in page 92 of [11]. This figure is the same as Figure $4 \mathrm{~B}$ above, and as stated in its legend, it has no competitive equilibrium. The comment [36] states that "limited arbitrage is satisfied in this case (Figure 7)", see last paragraph of [36]. However, as was shown in Example 2 above, the comment is wrong: Figure 7 in page 92 does not satisfy limited arbitrage. This is an example where there is no competitive equilibrium and limited arbitrage fails, and which is consistent with Theorem 1 in [11] and Theorem 2 above [16].

\section{Other Issues in [36]}

A number of other statements in [36] could confuse the reader.

1. [36] claims that "Chichilnisky's notion of gains from trade has no relevance for the existence of an equilibrium." 42 However, as shown in Proposition 2 and Corollary 1 above, and previously in [16], in Proposition 2 page 90 of [11] and mentioned in Proposition 1 of [14], p. 428, gains from trade are closely related to limited arbitrage, and therefore from Theorem 1 of [11] and Theorem 2 above and [16], to the existence of a competitive equilibrium. Limited arbitrage is necessary and sufficient for attainable and bounded gains from trade. ${ }^{43}$ The connection between limited arbitrage and bounded gains from trade is indeed very close.

2. [36] states ${ }^{44}$ that "One of the main objectives of Chichilnisky [14] and [8] appears to have been to obtain necessary and sufficient conditions for existence of equilibrium in terms of conditions limiting arbitrage for economic models in which agent's indifference surfaces are allowed to contain half lines". This statement contains two errors. The main purpose of my papers [14] and [8] was not to prove a necessary and sufficient condition for the existence of an equilibrium: [8] contains only results in algebraic topology and [14] contains only results in social choice. ${ }^{45}$ Of the three papers, the only one to contain details and proofs on equilibrium and limited arbitrage is [11], in Theorem $1 \mathrm{p}$. 94. Theorem 1 of [11] is correct exactly as stated and proved.

A second error in the statement quoted above is to imply that I focus on the case of

\footnotetext{
${ }^{42}$ See [36], page 1, first paragraph.

${ }^{43}$ See Proposition 2 above; in case (a) of [11] limited arbitrage is also necessary and sufficient for bounded gains from trade as stated in [14], and in Corollary 1 above.

${ }^{44}$ In para. 5 of its Introduction.

${ }^{45}$ Both of these papers refer the reader to [11] for details and proofs on equilibrium, see [14] p. 430, line 19 , and $[8]$ p. 195, lines 8-9.
} 
indifferences with half lines (a). This is incorrect: although case (b) is very simple I cover this case as well. ${ }^{46}$ Indeed my work on no-arbitrage and equilibrium in preferences without half lines goes back to 1984 [18] and [22]: results on no-arbitrage, the compactness of the Pareto frontier and the existence of a competitive equilibrium for preferences without half lines are in Chichilnisky and Heal [18] and in [22], Lemmas 4 and 5 and Theorem 1 .

3. In Section 5, under the title "Impact of Changes in Chichilnisky [11]", the comment states: "Below we argue that global cones in Chichilnisky [11] are not well defined".

It is easy to check that my global cones are well defined. In p. 84, Assumption 2, lines 3-5 of [11] I define two cases: " $\forall h:$ (a) the directions of the gradients of each indifference surface which is not bounded below define a closed set, or (b) indifferences contain no half lines". It is clear that whenever preferences have indifferences without half lines, we are in case (b). In [11], p. 84 (4) I define the global cone as follows: in case (a), the global cone is $A_{h}\left(\Omega_{h}\right)$ and in case (b), its closure $\overline{A_{h}}\left(\Omega_{h}\right)$.

The comment [36] mentions that cases (a) and (b) in Assumption 2 of [11] may overlap. ${ }^{47}$ This is irrelevant. The only possible overlap is when indifferences are bounded below and contain no half lines, but when preferences are all in this case any definition of the global cone $\left(A_{h}\right.$ or $\left.\bar{A}_{h}\right)$ works, because all my conditions and results hold true trivially. In this case limited arbitrage is always trivially satisfied (with either cone $A_{h}$ or $\bar{A}_{h}$ ), an equilibrium always exists, and the Pareto frontier is always compact because indifferences are bounded below.

\section{Conclusions}

The comment [36] contains several misunderstandings and errors.

- The example in the comment is a "mixed economy", while none of my three publications which the comment addresses-[8], [14] or [11]-claim to cover mixed economies. The comment is therefore irrelevant to the publications on which it comments.

- The publications on which the comment concentrates [8] and [14] contain brief announcements without details, and contain no proofs,

- Proofs of my results on equilibrium were published in [11]. These proofs are correct exactly as given and do not cover mixed economies. There is no inconsistency between the comment's example and any of my results, because they start from different conditions,

- Rather than being a counterexample, the example given in the comment [36] is a special case of my results for mixed economies of [16] reported above, results which the three authors acknowledge they have. Yet they failed to inform the reader of this consistency.

\footnotetext{
${ }^{46}$ Theorem 1 and Lemma 2 of [11] cover preferences with half lines in their indifferences as well as those without, see p. 84 of [11], Assumption 2, lines 3-4, where case (a) is with half lines and case (b) without balf lines. See also the definition of global cones for cases (a) and (b) in p. 85, section 2.2 of [11]; Lemma 2 p. 96 and 103 of [11] on the compactness of the Pareto frontier covers also cases (a) and (b), as does Theorem 1 on limited arbitrage being necessary and sufficient for the existence of a competitive equilibrium, p. 94 of [11].

${ }^{47}$ In paragraph 1 of the subsection on "impacts", Section 5.
} 
Furthermore in [11] I established:

- Global cones $G_{h}$ in [11] are well defined, and using these cones limited arbitrage bounds trades and utility exactly as needed for the existence of a competitive equilibrium,

- Limited arbitrage is necessary and sufficient for bounded and attainable gains from trade [11],

- Limited arbitrage is simultaneously necessary and sufficient for the existence of a competitive equilibrium and for the compactness of the Pareto frontier, and

- All the above results hold in economies with or without short sales, and whether traders' indifferences contain half lines or not.

The present paper:

- Extends all the above results to mixed economies which treat all preferences in a unified way, following [16],

- Defines social diversity and a topological invariant which contains exact information about the existence of a competitive equilibrium for the economy and its subeconomies,

- Shows that when limited arbitrage fails every quasiequilibrium has a failure of 'effective' demand.

\section{Appendix}

Proposition 3 Under Assumption 2: (i) The cone $G_{h}\left(\Omega_{h}\right)$ equals $A_{h}\left(\Omega_{h}\right)$ when indifferences contain half lines (case (a)) and its closure when they do not, (case (b)), and thus it is identical to the global cone defined in [11], (ii) the cones $\bar{A}_{h}\left(\Omega_{h}\right)=\bar{G}_{h}\left(\Omega_{h}\right)$ and are independent of initial endowments $\Omega_{h} \in R^{N}$ (iii) When preferences are in cases (a) and (b) the global cones $G_{h}\left(\Omega_{h}\right)$ are independent of initial endowments, but they are not necessarily independent in general, and (iv) None of these results need hold when Assumption 2 is not satisfied.

Proof. See also [16].

Define the sets:

$$
\begin{gathered}
B_{h}\left(\Omega_{h}\right)=\left\{x \in X: u_{h}\left(\Omega_{h}+\lambda x\right) \geq u_{h}\left(\Omega_{h}+\eta x\right) \text { when } \lambda \geq \eta \geq 0,\right. \\
\left.\lim _{\lambda_{j} \rightarrow \infty} u_{h}\left(\Omega_{h}+\lambda_{j} x\right)=u^{o}<\infty \text { and } \exists j: u_{h}\left(\Omega_{h}+\lambda_{j} x\right)=u^{o}\right\}
\end{gathered}
$$

and

$$
\begin{gathered}
C_{h}\left(\Omega_{h}\right)=\left\{x \in X: u_{h}\left(\Omega_{h}+\lambda x\right) \geq u_{h}\left(\Omega_{h}+\eta x\right) \text { when } \lambda \geq \eta \geq 0,\right. \\
\left.\lim _{\lambda_{j} \rightarrow \infty} u_{h}\left(\Omega_{h}+\lambda_{j} x\right)=u^{o}<\infty \text { and } \sim \exists j: u_{h}\left(\Omega_{h}+\lambda_{j} x\right)=u^{o}\right\} .
\end{gathered}
$$

The three sets $A_{h}\left(\Omega_{h}\right), B_{h}\left(\Omega_{h}\right)$ and $C_{h}\left(\Omega_{h}\right)$ are disjoint pairwise and

$$
A_{h}\left(\Omega_{h}\right) \cup B_{h}\left(\Omega_{h}\right) \cup C_{h}\left(\Omega_{h}\right) \cup H_{h}\left(\Omega_{h}\right)=R^{N} .
$$


where $H_{h}\left(\Omega_{h}\right)$ is the complement of $A_{h}\left(\Omega_{h}\right) \cup B_{h}\left(\Omega_{h}\right) \cup C_{h}\left(\Omega_{h}\right)$. Observe that by convexity of preferences $H_{h}\left(\Omega_{h}\right)$ is the set of directions along which the utility achieves a maximum value and decreases thereafter.

The first step is to observe that if $z \in B_{h}\left(\Omega_{h}\right) \cup C_{h}\left(\Omega_{h}\right)$, then for all $s \in R^{N}$

$$
s>z \Rightarrow s \in A_{h}\left(\Omega_{h}\right)
$$

and

$$
s<<z \Rightarrow s \in H_{h}\left(\Omega_{h}\right) .
$$

This follows from monotonicity and Assumption 2, which implies that the rate of increase of the utility is uniformly bounded below above zero along the direction defined by any strictly positive vector. Since the utility $u_{h}$ is non-satiated (8) and (9) together imply that for every $\varepsilon>0, \exists \lambda>0$ s.t. an $\varepsilon$ neighborhood of a vector $\lambda z \in B_{h}\left(\Omega_{h}\right) \cup C_{h}\left(\Omega_{h}\right)$ contains a vector $s$ in the set $A_{h}\left(\Omega_{h}\right)$ and another vector $v$ in the set $H_{h}\left(\Omega_{h}\right)$. This implies that the set $B_{h}\left(\Omega_{h}\right) \cup C_{h}\left(\Omega_{h}\right)$ is in the boundary of the set $A_{h}\left(\Omega_{h}\right)$. The relation between $G_{h}\left(\Omega_{h}\right)$ and $A_{h}\left(\Omega_{h}\right)$ stated in (i) is now immediate, cf. [11], p. 85, (4).

The next step is to show that if two different half-lines $l=\left\{\Omega_{h}+\lambda v\right\}_{\lambda>0}$ and $m=$ $\left\{\Lambda_{h}+\lambda v\right\}_{\lambda \geq 0}$ are parallel translates of each other, and $l \subset A_{h}\left(\Omega_{h}\right)$, then $m \subset A_{h}\left(\Lambda_{h}\right), \forall$ $\Lambda_{h} \in m$. This is immediate from Assumption 2, which ensures that the rate of increase of the utility is uniformly bounded above. Therefore the cone $A_{h}$ is independent of the initial endowments.

Observe that for a general convex preference represented by a utility $u_{h}$ the set $G_{h}\left(\Omega_{h}\right)$ itself may vary as the vector $\Omega_{h}$ varies, since the set $B_{h}\left(\Omega_{h}\right)$ itself may vary with $\Omega_{h}$; at some $\Omega_{h}$ a direction $z \in \partial G_{h}$ may be in $B_{h}\left(\Omega_{h}\right)$ and at others $B_{h}\left(\Omega_{h}\right)$ may be empty and $z \in C_{h}\left(\Omega_{h}\right)$ instead. This occurs when along a ray defined by a vector $z$ from one endowment the utility levels asymptote to a finite limit but do not reach their limiting value, while at other endowments, along the same direction $z$, they achieve this limit. This cannot happen when the preferences are in cases (a) or (b), but is otherwise consistent with Assumption 2. This example, and similar reasonings for $A_{h}\left(\Omega_{h}\right)$ completes the proof of the proposition.

Proof of Theorem 1 (Limited arbitrage is equivalent to the compactness of $U(\mathrm{E}))$ :

This result always holds when the consumption set is bounded below by some vector in the space, and in that case it is proved using standard arguments, see e.g. Arrow and Hahn [1]. Therefore in the following I concentrate in the case where $X$ is unbounded below.

Sufficiency first. Assume E has limited arbitrage. Since global cones are independent of initial endowments by Proposition 3, we may assume without loss of generality that $\Omega_{h}=0$ for all $h$. If $U(E)$ was not bounded there would exist a sequence of individually rational net trades $\left(z_{1}^{j} \ldots z_{H}^{j}\right)_{j=1,2 \ldots}$ and therefore correspondingly positive utility levels, such that: $\forall j, \sum_{h} z_{h}^{j}=0$, and for some $h, \lim _{j \rightarrow \infty} u_{h}\left(z_{h}^{j}\right)=\infty$. Therefore for some $h$, $\lim _{j}\left\|z_{h}^{j}\right\|=\infty$, and there exists a subsequence of its normalized vectors, denoted also by $\left\{z_{h}^{j} /\left\|z_{h}^{j}\right\|\right\}$, which is convergent. I will now prove that $z_{h}=\lim _{j} z_{h}^{j} /\left\|z_{h}^{j}\right\| \in \bar{G}_{h}$, the closure of $\bar{G}_{h}$. The proof is by contradiction. If $z_{h} \notin \bar{G}_{h}$ then by quasiconcavity of $u_{h}$ and by the proof of Proposition 3, along the ray defined by $z_{h}$ the utility $u_{h}$ achieves a maximum level $u^{o}$ at $\lambda_{o} z$, for $\lambda_{o} \geq 0$, and decreases thereafter: $\lambda>\lambda_{o} \Rightarrow u_{h}\left(\lambda z_{h}\right)<u^{o}$. Define a function $\theta: R_{+} \rightarrow R_{+}$by $u_{h}\left(\lambda z_{h}+\theta(\lambda) e\right)=u^{o}$, where $e=(1, \ldots, 1)$. I will show that $\theta$ is a convex function. By convexity of preferences,

$$
u^{o} \leq u_{h}\left(\alpha\left(\lambda z_{h}+\theta(\lambda) e\right)+(1-\alpha)\left(\hat{\lambda} z_{h}+\theta(\hat{\lambda}) e\right)\right)
$$




$$
=u_{h}\left((\alpha \lambda+(1-\alpha) \hat{\lambda}) z_{h}+(\alpha \theta(\lambda)+(1-\alpha)(\theta(\hat{\lambda})) e) .\right.
$$

Thus by monotonicity, $\theta(\alpha \lambda+(1-\alpha) \hat{\lambda}) \leq \alpha \theta(\lambda)+(1-\alpha) \theta(\hat{\lambda})$.

Assumption 2 together with monotonicity implies that the rate of increase of $u_{h}$ along the direction defined by $e$ (or by any strictly positive vector) is uniformly bounded below: $\exists \varepsilon>0:\left|u_{h}(x+\theta e)-u_{h}(x)\right| \geq|\theta| \varepsilon, \forall \theta \in R_{+}, \forall x \in R^{N}$. Therefore $u_{h}\left(\lambda z_{h}+\theta(\lambda) e\right) \equiv$ $u^{o} \geq u_{h}\left(\lambda z_{h}\right)+\theta(\lambda) \varepsilon$, so that $u_{h}\left(\lambda z_{h}\right) \leq u^{o}-\theta(\lambda) \varepsilon$. Note that $\theta\left(\lambda_{o}\right)=0$ and $\theta(\lambda)>0$ for $\lambda>\lambda_{0}$. Since $\theta$ is convex, $\lim _{\lambda \rightarrow \infty} \theta(\lambda)=\infty$, and $u_{h}\left(\lambda z_{h}\right) \leq u^{\circ}-\theta(\lambda) \varepsilon$ implies $u_{h}\left(\lambda z_{h}\right) \rightarrow-\infty$. It follows that $z_{h} \in \bar{G}_{h}$ for otherwise, since $\lim _{j}\left\|z_{h}^{j}\right\|=\infty$ and the vectors $z_{h}^{j}$ wander arbitrarily close to the direction defined by $z_{h}, \lim _{j \rightarrow \infty} u_{h}\left(z_{h}^{j}\right)<0$ contradicting individually rationality of the allocations $\left(z_{1}^{j} \ldots z_{H}^{3}\right)_{j=1,2 \ldots}$. Now recall that $\lim _{j \rightarrow \infty} u_{g}\left(z_{g}^{j}\right)=\infty$. By Assumption $2,\left|u_{g}(x)-u_{g}(y)\right| \leq K\|x-y\| \forall x, y \in R^{N}$, so for any $n$ and $j\left|u_{g}\left(z_{g}^{n}\right)-u_{g}\left(z_{g}^{n}-j e\right)\right| \leq K\|j e\|$, so for every $j$ there exists an $n_{j}$ such that $u_{g}\left(z_{g}^{n_{j}}-j e\right)>j$. Take the sequence $\left\{z_{g}^{n_{j}}\right\}$ and relabel it $\left\{z_{g}^{j}\right\}$. Now consider the new sequence of allocations $\left\{z_{1}^{j}+\frac{j e}{H-1}, \ldots, z_{g}^{j}-j e, \ldots, z_{H}^{j}+\frac{j e}{H-1}\right\}$ and call it also $\left\{z_{h}^{j}\right\}_{h=1,2, \ldots, H}$; this defines a feasible allocation for all $j$ and by Assumption $2, \forall h, u_{h}\left(z_{h}^{j}\right) \rightarrow \infty$. In particular $\left\|z_{h}^{j}\right\| \rightarrow \infty$. Define now $C$ as the set of all strictly positive convex combinations of the vectors $z_{h}$ for all $h$. Then either $C$ is strictly contained in a half space, or it defines a subspace of $R^{N}$. Since $\sum_{h} z_{h}^{j}=0, C$ cannot be strictly contained in a half space. Therefore. $C$ defines a subspace; in particular for any $g, \exists \lambda_{h} \geq 0 \forall h$ such that $\left({ }^{*}\right)$ $-z_{g} \equiv \sum_{h} \lambda_{h} z_{h}$. When one trader $g$ has indifferences without half lines $G_{g}=\bar{G}_{g}$ and $z_{g} \in \bar{G}_{g} \Rightarrow z_{g} \in G_{g}$, which by $\left({ }^{*}\right)$ contradicts limited arbitrage because $\sim \exists p:\left\langle p, z_{h}\right\rangle \geq 0$ for $z_{h} \in \bar{G}_{h}$ all $h$, and $\left\langle p, z_{g}\right\rangle>0 z_{g} \in G_{g}$. When for all $h$ the normalized gradients to any closed set of indifferent vectors defines a closed set, the global cone $G_{h}$ is open [11] so that its complement $G_{h}^{c}$ is closed, and the set of directions in $G_{h}^{c}$ is compact. On each direction of $G_{h}^{c}$ the utility $u_{h}$ achieves a maximum; therefore there exists for each $h$ a maximum utility level for $u_{h}$ over all directions in $G_{h}^{c}$. Since along the sequence $\left\{z_{h}^{j}\right\}$ every trader's utility increases without bound, $\forall h \exists j_{h}: j>j_{h} \Rightarrow z_{h}^{j} \in G_{h}$. However $\sum_{h} z_{h}^{j}=0$, again contradicting limited arbitrage. In all cases a contradiction arises from assuming that $U(\mathrm{E})$ is not bounded, so $U(\mathrm{E})$ must be bounded.

The next step is to prove that $U(\mathrm{E})$ is closed when limited arbitrage is satisfied. Consider a sequence of allocations $\left\{z_{h}^{j}\right\}_{j=1,2 \ldots}, h=1,2, \ldots, H$, satisfying $\forall j, \sum_{h=1}^{H} z_{h}^{j}=0$. Assume that $\forall j, u_{1}\left(z_{1}^{j}\right), \ldots, u_{H}\left(z_{H}^{3}\right) \subset R_{+}^{H}$ and converges as $j \rightarrow \infty$ to a utility allocation $v=\left(v_{1}, \ldots, v_{H}\right) \in R_{+}^{H}$, which is undominated by any other feasible utility allocation. Observe that the vector $v$ may or may not be the utility vector of a feasible allocation: when limited arbitrage is satisfied, I will prove that it is. The result is immediate if the sequence of allocations $\left\{z_{h}^{j}\right\}_{j=1,2 \ldots}, h=1,2, \ldots, H$ is bounded; therefore I concentrate in the case where the set of feasible allocations is not bounded. Let $M$ be the set of all traders $h \in\{1,2 .,,, H\}$, which may be empty, for whom the corresponding sequence of allocations $\left\{z_{h}^{j}\right\}_{j=1,2 \ldots}$ is bounded, i.e. $h \in M \Leftrightarrow \exists K_{h}: \forall j,\left\|z_{h}^{j}\right\|<K_{h}<\infty$; let $J$ be its complement, $J=\{1,2, \ldots, H\}-M$, which $\mathrm{I}$ assume to be non empty. There exists a subsequence of the original sequence of allocations, which for simplicity is denoted also $\left\{z_{h}^{j}\right\}_{j=1,2 \ldots}$, $h=1,2, \ldots, H$, along which $\forall h \in M, \lim _{j}\left\{z_{h}^{j}\right\}_{j=1,2 \ldots}=z_{h}$ exists, and by construction $\sum_{h \in M} z_{h}+\lim _{j \rightarrow \infty} \sum_{h \in J} z_{h}^{j}=0$. Consider now the sequence $z_{h}^{j} /\left\|z_{h}^{j}\right\|$ for $h \in J$; it has a convergent subsequence, denoted also $z_{h}^{j} /\left\|z_{h}^{j}\right\|$. Define $z_{h}=\lim _{j} z_{h}^{j} /\left\|z_{h}^{j}\right\|, h \in J$. Then as seen in the first part of this proof, $\forall h \in J, z_{h} \in \bar{G}_{h}(0)$, because $\left\|z_{h}^{j}\right\| \rightarrow \infty$ and $u_{h}\left(z_{h}^{j}\right) \geq 0$. If $\forall h \in J, z_{h} \notin G_{h}(0)$ then the utility values of the traders attain their limit for all $h$ and the utility vector $v$ is achieved by a feasible allocation. Therefore the proof 
is complete. It only remains to consider the case where for some trader $g \in J, z_{g} \in G_{g}(0)$. As above, let $C$ be the convex cone of all strictly positive linear combinations of the vectors $\left\{z_{h}\right\}_{h \in J}$. Then either $C$ is contained strictly in a half-space of $R^{N}$, or $C$ spans a subspace of $R^{N}$. Since $\sum_{h \in M} z_{h}+\lim _{j \rightarrow \infty} \sum_{h \in J} z_{h}^{j}=0, C$ cannot be strictly contained in a half space, for otherwise $\lim _{j \rightarrow \infty} \sum_{h \in J} z_{h}^{j}$ would not be bounded as $\forall h \in J,\left\|z_{h}^{j}\right\| \rightarrow \infty$. Therefore there exists a subspace $S \subset R^{N}$ spanned by $\left\{z_{h}\right\}_{h \in J}$. In particular, $-z_{g} \in S$, i.e. $\forall h \in J, \exists \lambda_{h} \geq 0$ such that $\left(^{*}\right)-z_{g}=\sum_{h \in J} \lambda_{h} z_{h}$. Since in this last case $\forall h \in J$, $z_{h} \in \overline{G_{h}}(0)$ and for $h=g, z_{g} \in G_{g}(0)$, by limited arbitrage $\exists p \in \cap_{h} D_{h}$ s. t. $\left\langle p, z_{g}\right\rangle>0$, and $\forall h,\left\langle p, z_{h}\right\rangle \geq 0$, which contradicts $\left(^{*}\right)$. Since the contradiction arises from assuming that the set $U(\mathrm{E})$ is not closed, $U(\mathrm{E})$ must be closed. Thus $U(\mathrm{E})$ is compact. In particular, since $P(\mathrm{E})$ is the boundary of $U(\mathrm{E})$, limited arbitrage implies a compact Pareto frontier $P(\mathrm{E})$.

Necessity is established next. If limited arbitrage fails, there is no vector $y \in R^{N}$ such that $\left\langle y, z_{h}\right\rangle>0$ for all $\left\{z_{h}\right\} \in G_{h}(0)$. Equivalently, there exist a set $J$ consisting of at least two traders and, for each $h \in J$, a vector $z_{h} \in G_{h}(0)$ such that $\sum_{h \in J} z_{h}=0$. Then either for some $h, z_{h} \in A_{h}$ so that the Pareto frontier is unbounded and therefore not compact, or else for some $h, z_{h} \in \partial G_{h}(0) \cap G_{h}(0)$ and therefore the Pareto frontier is not closed, and therefore $U(\mathrm{E})$ is not compact. In either case, the Pareto frontier is not compact when limited arbitrage fails. Therefore compactness of $U(\mathrm{E})$ is necessary for limited arbitrage.

Proof of Theorem 2 (Limited arbitrage is equivalent to the existence of a competitive equilibrium):

Necessity first. Consider first the case $X=R^{N}$ and assume without loss of generality that $\Omega_{h}=0$ for all $h$. The proof is by contradiction. Assume that limited arbitrage fails and let $p^{*}$ be an equilibrium price and $x^{*}=\left(x_{1}^{*}, \ldots x_{H}^{*}\right)$ the corresponding equilibrium allocation. A failure of limited arbitrage means that $\exists\left(z_{1}, \ldots, z_{H}\right): \forall h, z_{h} \in G_{h}(0)$, and $\sum_{h=1}^{H} z_{h}=0$. By construction $u_{h}\left(\lambda z_{h}\right)$ never ceases to increase with $\lambda$, because $z_{h} \in G_{h}(0)$. Since global cones are uniform $x_{h}^{*}+z_{h} \in G_{h}\left(x_{h}^{*}\right)$, so that by the same reasoning $x^{*}$ cannot be Pareto efficient, contradicting the fact that $x^{*}$ is a competitive equilibrium

Consider next $X=R_{+}^{N}$. Assume that $\forall q \in S(\mathrm{E}) \exists h \in\{1, \ldots, H\}$ such that $\left\{q, \Omega_{h}\right\rangle=0$. Then if limited arbitrage is not satisfied $\bigcap_{h=1}^{H} D_{h}^{+}\left(\Omega_{h}\right)=\emptyset$, which implies that $\forall p \in S(\mathrm{E})$, $\exists h$ and $v(p) \in G_{h}\left(\Omega_{h}\right)$ :

$$
\langle p, \lambda v(p)\rangle \leq 0, \forall \lambda>0
$$

This implies that a competitive equilibrium price cannot exist. By contradiction. Let $p^{*}$ be an equilibrium price and $x^{*} \in X^{H}$ be the corresponding equilibrium allocation. Then $p^{*} \in S(E)$, so that by $(12) x_{h}^{*}+\lambda v(p)$ is affordable and strictly preferred to $x^{*}$ for some $\lambda>0$, contradicting the assumption that $x^{*}$ is an equilibrium allocation. Therefore limited arbitrage is also necessary for the existence of a competitive equilibrium in this case.

It remains to consider the case where $\exists p \in S(\mathrm{E})$ such that $\forall h \in\{1, \ldots, H\},\left\langle p, \Omega_{h}\right\} \neq 0$. But in this case by definition $\bigcap_{h=1}^{H} D_{h}^{+}\left(\Omega_{h}\right) \neq \emptyset$ since $\forall h \in\{1 \ldots H\}, R_{++}^{N} \subset D_{h}^{+}\left(\Omega_{h}\right)$, so that limited arbitrage is always satisfied when an equilibrium exists. This completes the proof of necessity when $X=R_{+}^{N}$.

Sufficiency next. The proof uses the fact that the Pareto frontier is homeomorphic to a simplex. When $X=R_{+}^{N}$ the Pareto frontier of the economy $P(\mathrm{E})$ is always homeomorphic to a simplex, see Arrow and Hahn [1]. In the case $X=R^{N}$ this may fail: for example $U(E)$ may be unbounded. However, by Theorem 1 above, if the economy satisfies limited 
arbitrage then the utility possibility set $U(\mathrm{E}) \subset R_{+}^{N}$ and the Pareto frontier $P(\mathrm{E}) \subset R_{+}^{N}$ are compact; under the assumptions on preferences, $P(E)$ is then also homeomorphic to a simplex [1]. Therefore in both cases, $P(\mathrm{E})$ is homeomorphic to a simplex and I can apply a method due to Chichilnisky and Heal [18] which extends Negishi's method of using a fixed point argument on the Pareto frontier ${ }^{48}$ to establish the existence of a pseudoequilibrium with or without short sales. ${ }^{49}$ It remains however to prove that the pseudoequilibrium is also a competitive equilibrium.

To complete the proof of existence of a competitive equilibrium consider first $X=$ $R^{N}$. Then $\forall h=1, \ldots, H$ there exists an allocation in $X$ of strictly lower value than the pseudoequilibrium $x_{h}^{*}$ at the price $p^{*}$. Therefore by Lemma 3, Chapter 4, page 81 of Arrow and Hahn [1], the quasi-equilibrium $\left(p^{*}, x^{*}\right)$ is also a competitive equilibrium, completing the proof of existence when $X=R^{N}$.

Next consider $X=R_{+}^{N}$, and a quasi-equilibrium $\left(p^{*}, x^{*}\right)$ whose existence was already established. If every individual has a positive income at $p^{*}$, i.e. $\forall h,\left\langle p^{*}, \Omega_{h}\right\rangle>0$, then by Lemma 3, Chapter 4 of Arrow and Hahn [1] the quasi-equilibrium $\left(p^{*}, x^{*}\right)$ is also a competitive equilibrium, completing the proof. Furthermore, observe that in any case the pseudoequilibrium price $p^{*} \in S(\mathrm{E})$, so that $S(\mathrm{E})$ is not empty. To prove existence we consider therefore two cases: first the case where $\exists q^{*} \in S(\mathrm{E}): \forall h,\left\langle q^{*}, \Omega_{h}\right\rangle>0$. In this case, by the above remarks from Arrow and Hahn [1], $\left(q^{*}, x^{*}\right)$ is a competitive equilibrium. The second case is when $\forall q \in S(\mathrm{E}), \exists h \in\{1, \ldots, H\}$ such that $\left\langle q, \Omega_{h}\right\rangle=0$. Limited arbitrage then implies:

$$
\exists q^{*} \in S(\mathrm{E}): \forall h,\left\langle q^{*}, v\right\rangle>0 \text { for all } v \in G_{h}\left(\Omega_{h}\right) .
$$

Let $x^{*}=x_{1}^{*}, \ldots, x_{H}^{*} \in X^{H}$ be a feasible allocation in $\Upsilon$ supported by the vector $q^{*}$ defined in (13): by definition, $\forall h, u_{h}\left(x_{h}^{*}\right) \geq u_{h}\left(\Omega_{h}\right)$ and $q^{*}$ supports $x^{*}$. Note that any $h$ minimizes costs at $x_{h}^{*}$ because $q^{*}$ is a support. Furthermore $x_{h}^{*}$ is affordable under $q^{*}$. Therefore, $\left(q^{*}, x^{*}\right)$ can fail to be a competitive equilibrium only when for some $h,\left\langle q^{*}, x_{h}^{*}\right\rangle=0$, for otherwise the cost minimizing allocation is always also utility maximizing in the budget set $B_{h}\left(q^{*}\right)=\left\{w \in X:\left\langle q^{*}, w\right\rangle=\left\langle q^{*}, \Omega_{h}\right\rangle\right\}$.

It remains therefore to prove existence when $\left\langle q^{*}, x_{h}^{*}\right\rangle=0$ for some $h$. Since by the definition of $S(\mathrm{E}), x^{*}$ is individually rational, i.e. $\forall h, u_{h}\left(x_{h}^{*}\right) \geq u_{h}\left(\Omega_{h}\right)$, then $\left\langle q^{*}, x_{h}^{*}\right\rangle=0$ implies $\left\langle q^{*}, \Omega_{h}\right\rangle=0$, because by definition $q^{*}$ is a supporting price for the equilibrium allocation $x^{*}$. If $\forall h, u_{h}\left(x_{h}^{*}\right)=0$ then $x_{h}^{*} \in \partial R_{+}^{N}$, and by the monotonicity and quasiconcavity of $u_{h}$, any vector $y$ in the budget set defined by the price $p^{*}, B_{h}\left(q^{*}\right)$, must also satisfy $u_{h}(y)=0$, so that $x_{h}^{*}$ maximizes utility in $B_{h}\left(q^{*}\right)$, which implies that $\left(q^{*}, x^{*}\right)$ is a competitive equilibrium. Therefore $\left(q^{*}, x^{*}\right)$ is a competitive equilibrium unless for some h. $u_{h}\left(x_{h}^{*}\right)>0$.

Assume therefore that the quasiequilibrium $\left(q^{*}, x^{*}\right)$ is not a competitive equilibrium, and that for some $h$ with $\left\langle q^{*}, \Omega_{h}\right\rangle=0, u_{h}\left(x_{h}^{*}\right)>0$. Since $u_{h}\left(x_{h}^{*}\right)>0$ and $x_{h}^{*} \in \partial R_{+}^{N}$ then an indifference surface of a commodity bundle of positive utility $u_{h}\left(x_{h}^{*}\right)$ intersects $\partial R_{+}^{N}$ at $x_{h}^{*} \in \partial R_{+}^{N}$. Let $r$ be the ray in $\partial R_{+}^{N}$ containing $x_{h}^{*}$. If $w \in r$ then $\left\langle q^{*}, w\right\rangle=0$, because $\left\langle q^{*}, x_{h}^{*}\right\rangle=0$. Since $u_{h}\left(x_{h}^{*}\right)>0$, by Assumption $1 u_{h}$ does not reach a maximum along $r$, so that $w \in G_{h}\left(x_{h}^{*}\right)$. But this contradicts the choice of $q^{*}$ as a supporting price satisfying

\footnotetext{
${ }^{48} \mathrm{Negishi}$ 's method for proving the existence of a pseudoequilibrium [37] applies only to the case where the economy has no short sales. This result was extended by Chichilnisky and Heal [18] and [22] to economies with short sales, where feasible desirable allocations may be unbounded, see also [20] and Chichilnisky [11].

${ }^{49} \mathrm{~A}$ pseudoequilibrium, also called quasiequilibrium, is an allocation and a price at which traders minimize cost and markets clear.
} 
limited arbitrage (13) since

$$
\exists h \text { and } w \in G_{h}\left(\Omega_{h}\right) \text { such that }\left\langle q^{*}, w\right\rangle=0 .
$$

The contradiction between (14) and (13) arose from the assumption that $\left(q^{*}, x^{*}\right)$ is not a competitive equilibrium, so that $\left(q^{*}, x^{*}\right)$ must be a competitive equilibrium, and the proof is complete.

\section{References}

[1] Arrow, Kenneth, and Frank Hahn (1971) General Competitive Analysis, North Holland, 1986, San Francisco and New York.

[2] Arrow, K. and G. Debreu (1954) "Existence of an Equilibrium for a Competitive Economy" Econometrica 22, p. 264-90.

[3] Arrow, Kenneth (1994) Report on mediation: "My interpretation of a disputed history." A vailable from the author upon request, December 28, 1994.

[4] Chichilnisky, Graciela "Manifolds of Preferences and Equilibria" (1976) Ph.D. Dissertation, Department of Economics, University of California, Berkeley.

[5] Chichilnisky, Graciela (1980) "Intersecting Families of Sets" Working Paper, University of Essex, U.K.

[6] Chichilnisky, Graciela (1986) "Topological Complexity of Manifolds of Preferences" Chapter 8, Essays in Honor of Gerard Debreu (W. Hildenbrand and A. MasColell eds.) North-Holland, New York, p. 131-142.

[7] Chichilnisky, Graciela (1991) "Markets, Arbitrage and Social Choice", presented at the conference "Columbia Celebrates Arrow's Contributions" Columbia University, New York, October 27, 1991, Working Paper No. 586 Columbia University, Department of Economics, December 1991, and C.O.R.E. Discussion Paper No. 9342, CORE Universite Catolique de Louvain, Louvain la Neuve, Belgium, 1993.

[8] Chichilnisky, Graciela (1993) "Intersecting Families of Sets and the Topology of Cones in Economics" Bulletin of the American Mathematical Society, October 1993, Vol. 29, No. 2, p. 189-207.

[9] Chichilnisky, Graciela (1993) "Limited Arbitrage is Necessary and Sufficient for the Existence of the Core" Working Paper, Columbia University, revised 1994.

[10] Chichilnisky, Graciela (1992) "Limited Arbitrage is Necessary and Sufficient for the Existence of a Competitive Equilibrium", Working Paper No. 650, Columbia University, December 1992.

[11] Chichilnisky, Graciela (1995) "Limited Arbitrage is Necessary and Sufficient for the Existence of a Competitive Equilibrium With or Without Short Sales", Economic Theory, Vol. 5, No. 1, p. 79-108, January 1995.

[12] Chichilnisky, Graciela (1994) "Limited Arbitrage is Necessary and Sufficient for the Existence of a Competitive Equilibrium and the Core and it Limits Voting Cycles", Working Paper, Fall 1993, presented and distributed at the Yearly Meetings of 
the American Economic Association, Boston, January 3-5, 1994, Economic Letters, Vol. 46, December 1994, p. 321-331. The volume was reprinted in 3/95 without the paper, which is due to reappear under the title "Limited Arbitrage is Necessary and Sufficient for the Nonemptiness of the Core" in Economic Letters, 1996.

[13] Chichilnisky, Graciela (1991) "Limited Arbitrage and Social Diversity: Markets and Social Choices", Social Choice and Welfare, forthcoming.

[14] Chichilnisky, Graciela (1992) "Limited Arbitrage, Gains from Trade and Social Diversity: A Unified Perspective on Resource Allocation" Working Paper Columbia University, American Economic Review, Vol. 84, No. 2, May 1994, p. 427-434.

[15] Chichilnisky, Graciela (1993) "Topology and Economics: the Contribution of Stephen Smale", From Topology to Computation, Proceedings of the Smalefest (M. Hirsch, J. Marsden, and M. Shub. eds.) Springer Verlag, New York-Heidelberg, p. 147-161.

[16] Chichilnisky, Graciela (1995) "A Unified Perspective on Resource Allocation: Limited Arbitrage is Necessary and Sufficient for the Existence of a Competitive Equilibrium, the Core, and Social Choice" CORE Discussion Paper No. 9527, Universite Catolique de Louvain, April 1995, revised November 1995 and March 1996. Invited presentation at the International Economics Association Round Table on Social Choice, Vienna, May 1994, a revised version forthcoming in Social Choice Reexamined, (K. Arrow, A. Sen and T. Suzumura) MacMillan, 1996.

[17] Chichilnisky, Graciela (1995) "Concave Preferences in Finite or Infinite Dimensions" Working Paper, Columbia University, 1995.

[18] Chichilnisky, Graciela and Geoffrey M. Heal (1984) "Existence of a Competitive Equilibrium in $L_{p}$ and Sobolev Spaces", IMA Preprint series No. 79, Institute for Mathematics and its Applications, University of Minnesota, Minneapolis, Minnesota, June 1984.

[19] Chichilnisky, Graciela and Geoffrey M. Heal (1991) "Arbitrage and the Pareto Frontier" Working Paper, Columbia Business School.

[20] Chichilnisky, Graciela and Geoffrey M. Heal (1991) "Arbitrage and Equilibrium in Sobolev Spaces" First Boston Paper Series 92-29, July 1992, revised in February 1995 under the title: "Equilibrium and the Core with Finitely or Infinitely Many Markets: A Unified Approach"

[21] Chichilnisky, Graciela and Geoffrey M. Heal (1992) "Arbitrage and Equilibrium with Infinitely Many Securities and Commodities" Discussion Paper Series No. 618, Columbia University Department of Economics, July 1992.

[22] Chichilnisky, Graciela and G. M. Heal (1993) "Existence of a Competitive Equilibrium in Sobolev Spaces without Bounds on Short Sales", Journal of Economic Theory, Vol. 59, No. 2, p. 364-384.

[23] Debreu, Gerard (1959) The Theory of Value, Cowles Foundation Monograph, John Wiley, New York. 
[24] Debreu, Gerard (1962) "New Concepts and Techniques in Equilibrium Analysis", International Economic Review, Vol 3, p. 257-73.

[25] Grandmont, Jean Michel (1982) "Temporary Equilibrium" Chapter in Handbook of Mathematical Economics, (eds. K. Arrow and M. Intriligator) North Holland, New York.

[26] Green, Jerry (1973) "Temporary Equilibrium in a Sequential Trading Model with Spot and Futures Transactions" Econometrica, Vol. 41, No. 6, p. 1103 - 23.

[27] Hammond, Peter (1983) "Overlapping Expectations and Hart's Conditions for Equilibrium in a Securities Market" Journal of Economic Theory, 31, p. 170-75.

[28] Hart, Oliver (1974) "Existence of Equilibrium in a Securities Model" Journal of Economic Theory, 9, p. 293-311.

[29] Heller, W., M. Hirsch, T. Groves, W. Shafer and others (1996), Letter to P. Monteiro, F. Page and M. Wooders, January 10, 1996. Available from the author upon request.

[30] Heller, W., M. Hirsch (1996) Letter to F. Page and M. Wooders, February 29, 1996. Available from the author upon request.

[31] Kreps, David (1981) "Arbitrage and Equilibrium in Economies with Infinitely Many Commodities" Journal of Mathematical Economics, Vol. 8, p. 15-35.

[32] McKenzie, Lionel (1959) "On the existence of a general equilibrium for competitive markets" Econometrica, 27, p. 54-71.

[33] McKenzie, Lionel (1987) "General Equilibrium", Chapter 1, General Equilibrium, The New Palgrave (eds. J. Eatwell, M. Milgate, P. Newman) Norton, New York.

[34] McKenzie, Lionel (1961) "On the Existence of General Equilibrium: Some Corrections" Econometrica, 29, p. 247-248.

[35] Koutsougeras, Leonid (1993) "The core in two-stage games" Working Paper, University of Illinois, Urbana, Illinois. CORE Discussion Paper No. 9525, Universite Catolique de Louvain.

[36] Monteiro, P., F. Page and M. Wooders (1995) "Arbitrage, Equilibrium and Gains from Trade: A Counterexample" this issue of Journal of Mathematical Economics.

[37] Negishi, T. (1960) "Welfare Economics and the Existence of an Equilibrium for a Competitive Economy" Metroeconomica 12, p. 92-97.

[38] Nielsen, Lars (1989) "Asset Market Equilibrium with Short Selling" Review of Economic Studies, Vol. 56., No. 187, p. 467-473.

[39] Page, Frank (1987) "Notes and Comments to the Editor: On Equilibrium in Hart's Securities Exchange Model" Journal of Economic Theory, 41, p. 392-404.

[40] Spanier, Edwin (1979) Algebraic Topology, McGraw Hill, New York.

[41] Werner, Jan (1987) "Arbitrage and the Existence of Competitive Equilibrium", Econometrica 55, No. 6, p. 1403-1418. 


\title{
1995-1996 Discussion Paper Series
}

\author{
Department of Economics \\ Columbia University \\ 1022 International Affairs Bldg. \\ 420 West 118th Street \\ New York, N.Y., 10027
}

The following papers are published in the 1995-96 Columbia University Discussion Paper series which runs from early November to October 31 of the following year (Academic Year).

Domestic orders for discussion papers are available for purchase at the cost of $\$ 8.00$ (U.S.) Per paper and $\$ 140.00$ (US) for the series.

Foreign orders cost $\$ 10.00$ (US) per paper and $\$ 185.00$ for the series.

To order discussion papers, please write to the Discussion Paper Coordinator at the above address along with a check for the appropriate amount, made payable to Department of Economics, Columbia University. Please be sure to include the series number of the requested paper when you place an order. 


\section{5-96 Discussion Papers Series}

9596-01 Protectionist Response to Import Competition in Declining Industries Reconsidered

9596-02 New Estimates on Climate Demand: Evidence from Location Choice

9596-03 Enforcement by Hearing

9596-04 Preferential Trading Areas and Multilateralism: Strangers, Friends or Foes?

9596-05 Simplification, Progression and a Level Playing Field

9596-06 The Burden of Proof in Civil Litigation

9596-07 Market Structure and the Timing of Technology Adoption

9596-08 The Emergence of the World Economy

9596-09 The Global Age: From a Skeptical South to a Fearful North

9596-10 A Conformity Test for Cointegration

9596-11 Identification and Kullback Information in the GLSEM

9596-12 Informational Leverage and the Endogenous Timing of Product Introductions

9596-13 Changes in Wage Inequality

9596-14 The Design of Monte Carlo Experiments for VAR Models

9596-15 A Toplogical Invariant for Competitive Markets by: J. Choi

by: M. Cragg

M. Kahn

by: C. Sanchirico

by: J. Bhagwati

A. Panagariya

by: W. Vickrey

by: C. Sanchirico

by: J. Choi

M. Thum

by: R. Findlay

by: J. Bhagwati

by: P. Dhrymes

by: P. Dhrymes

by: J. Choi

by: J. Mincer

by: P. Dhrymes

by: G. Chichilnisky 